\title{
Close and ordinary social contacts: How important are they in promoting large-scale contagion?
}

\author{
Peng-Bi Cui (崔鹏碧), ${ }^{1,2,3}$ Wei Wang, ${ }^{4}$ Shi-Min Cai, ${ }^{1,2,3, *}$ Tao Zhou, ${ }^{1,3,2}$ and Ying-Cheng Lai ${ }^{5}$ \\ ${ }^{1}$ School of Computer Science and Engineering, University of Electronic Science and Technology of China, Chengdu 610054, China \\ ${ }^{2}$ Institute of Fundamental and Frontier Science, University of Electronic Science and Technology of China, Chengdu 610073, China \\ ${ }^{3}$ Big Data Research Center, University of Electronic Science and Technology of China, Chengdu 611731, China \\ ${ }^{4}$ Cybersecurity Research Institute, Sichuan University, Chengdu 610065, China \\ ${ }^{5}$ School of Electrical, Computer, and Energy Engineering, Arizona State University, Tempe, Arizona 85287-5706, USA
}

(Received 28 June 2018; revised manuscript received 6 October 2018; published 28 November 2018)

\begin{abstract}
An outstanding problem of interdisciplinary interest is to understand quantitatively the role of social contacts in contagion dynamics. In general, there are two types of contacts: close ones among friends, colleagues and family members, etc., and ordinary contacts from encounters with strangers. Typically, social reinforcement occurs for close contacts. Taking into account both types of contacts, we develop a contact-based model for social contagion. We find that, associated with the spreading dynamics, for random networks there is coexistence of continuous and discontinuous phase transitions, but for heterogeneous networks the transition is continuous. We also find that ordinary contacts play a crucial role in promoting large-scale spreading, and the number of close contacts determines not only the nature of the phase transitions but also the value of the outbreak threshold in random networks. For heterogeneous networks from the real world, the abundance of close contacts affects the epidemic threshold, while its role in facilitating the spreading depends on the adoption threshold assigned to it. We uncover two striking phenomena. First, a strong interplay between ordinary and close contacts is necessary for generating prevalent spreading. In fact, only when there are propagation paths of reasonable length which involve both close and ordinary contacts are large-scale outbreaks of social contagions possible. Second, abundant close contacts in heterogeneous networks promote both outbreak and spreading of the contagion through the transmission channels among the hubs, when both values of the threshold and transmission rate among ordinary contacts are small. We develop a theoretical framework to obtain an analytic understanding of the main findings on random networks, with support from extensive numerical computations. Overall, ordinary contacts facilitate spreading over the entire network, while close contacts determine the way by which outbreaks occur, i.e., through a second- or first-order phase transition. These results provide quantitative insights into how certain social behaviors can emerge and become prevalent, which has potential implications not only to social science but also to economics and political science.
\end{abstract}

DOI: 10.1103/PhysRevE.98.052311

\section{INTRODUCTION}

The spreading of certain behaviors (contagion) in the human society has social, economical, and political implications, which has attracted a great deal of interdisciplinary research effort. Conventional methods developed to uncover and understand the dynamics of social contagion are more or less based on some kind of threshold and memory effects [1-10]. For example, it is likely for an individual to adopt certain behavior if he/she possesses friends who have already adopted the behavior. From a network point of view, for a node to adopt certain behavior, the number of connected nodes who have already accepted and exhibit the behavior must exceed a threshold [1,11]. That is, an individual will become willing to adopt a behavior if he/she has received sufficient and repeated information about the behavior from his/her friends (or neighbors in the underlying social network) $[12,13]$. In the real world, the influences of network neighbors and the tendency for any individual to adopt certain behavior can be

\footnotetext{
*shimin.cai81@gmail.com
}

highly nonuniform. There is empirical evidence that individuals and their social contacts tend to play heterogeneous roles in contagion $[14,15]$. For example, regardless of the nature of the behavior, there always exist certain individuals who are reluctant to accept or adopt the behavior. To account for the heterogeneity, a variant of the classic threshold model [1] was introduced [11], where a certain fraction of nodes were assumed to be immune to the behavior. These are the blocked nodes, and if their number or density is large enough, both the extent and diffusion speed of the contagion spreading will be suppressed, provided that no cascading or avalanche type of processes occur. The effects of heterogeneity in social contacts have been recently studied [16] using a contagion threshold model incorporating weighted edges, with the finding that heterogeneity in the weights can suppress the propagation of contagion.

The influences from connected neighbors in a social network represent a kind of reinforcement effect, where the probability of adopting certain social behavior by an individual is modified when he/she receives information about the behavior through social contacts. In recent years, spreading dynamics driven by reinforcement have attracted a great deal of attention 
from researchers in diverse fields including social science, economics, and physics [9,12,17-20]. The key fact that motivated our present work is that, with respect to reinforcement, the nature of social contacts can have drastically different impacts. In particular, from close social contacts such as family members, friends, and colleagues, reinforcement can be much stronger than that from conventional or ordinary social contacts with, e.g., strangers. For a model of social contagion to capture the real behaviors as accurately as possible, the distinct reinforcement effects from close and ordinary social contacts must be taken into account.

The need to distinguish two types of social contacts in terms of their influences has been well documented in the literature. Historically, Lazarsfeld and Merton pointed out that mass media messages can be reinforced by interpersonal communications [21]. There were empirical evidence and theories for the conjecture that more interpersonal conversation or discussions can promote the impact of media information through reinforcement [22,23]. In general, individuals are more likely to engage in interpersonal communications with family, friends or colleagues as often as they get media news or messages from newspapers or other individuals [24]. That is, for reinforcement, close social contacts are more effective than interaction with machines or strangers. At the present, there exists considerable empirical evidence that, even for interpersonal communications, there is heterogeneity in their role in social reinforcement. In general, the degree of reinforcement depends on factors such as individual responsiveness, the number of neighbors capable of reinforcing, and respondent-reinforcer pairing [25-27]. For example, the number of reinforcers is determined by the degree of the individual in the social network, and reinforcement among strangers, even if there are social contacts among them, is far less likely than that among close relationships [27]. That is, in reality, social contagion is strongly contact-based, and not all social contacts can lead to reinforcement.

To our knowledge, in the current literature, there is no work on social contagion dynamics which takes into account the two distinct types of social contacts: close and ordinary. In general, both types of contacts exist, and the question is how their coexistence affects the basic dynamical behaviors. In this paper, we propose a general model of social contagion with two distinct transmission channels: one through close and another through ordinary contacts. For random networks, we develop an analytical edge-based compartmental method to solve the model, which enables us to make a number of predictions in terms of the fundamental characterizing quantities such as the final outbreak size, the outbreak threshold, and the nature of the phase transition. We also carry out extensive agent-based, stochastic simulations to assess the performance of the model. For random networks, the computations reveal that continuous (second order) and discontinuous (first order) phase transitions coexist in random networks, which is ascertained by an analytic bifurcation analysis of the system. A computational study of two representative empirical networks with a heterogeneous degree distribution from the real world reveals that only continuous transitions can be expected. We provide a physical understanding of the basic spreading dynamics through a detailed statistical analysis, uncover the conditions under which contagion prevalence can arise, and validate the existence of an optimal fraction of ordinary contacts for outbreak at a global scale.

Two striking phenomena are uncovered. One is that an interplay between ordinary and close contacts is necessary for generating prevalent spreading on random networks. In particular, only when there are propagation paths of sufficient length which involve both close and ordinary contacts are largescale outbreaks of social contagions possible. The second phenomenon is that, for heterogeneous networks, a considerable number of close contacts will promote outbreak of the contagion by forming channels for successful transmissions among hubs. Taken together, ordinary contacts make possible spreading over the entire network, while close contacts not only shape the way by which outbreaks occur, i.e., through a second or first order phase transition in random networks, but also facilitate local spreading and outbreaks in heterogeneous networks. These results provide quantitative insights into how social behaviors can emerge and become prevalent, which has potential implications not only to social science, but also to economics and political science.

In Sec. II, we present our general model of spreading dynamics with two distinct types of social contacts. In Sec. III, we describe the edge-based compartmental analysis approach. In Sec. IV, we implement our spreading model on random networks and carry out agent-based simulations and a comprehensive theoretical analysis, and then extend our study to empirical heterogeneous networks. In Sec. V, we present conclusions and an outlook.

\section{MODEL}

To gain basic insights into the roles of close and ordinary contacts in social contagion dynamics, we first assume that the individual social relationships are described by a random network of size $N$ (i.e., Erdös-Rényi (ER) networks), where a pair of nodes are connected with each other with probability $p_{e}$ so that the network degree distribution and the mean degree are $p(k)=e^{-\langle k\rangle}\langle k\rangle^{k} / k$ ! and $\langle k\rangle=N p_{e}$, respectively. The total number of edges is $E=N\langle k\rangle / 2$, where each edge represents a particular social contact between two individuals. The dynamics of social contagion are governed by the standard susceptible-adopted-recovered (SAR) model, where any node (individual) can be in one of the three states. In particular, a node in the susceptible state may adopt a behavior when it receives information about it from its neighbors who have already adopted the behavior. If a node is in the adopted state, then with certain probability it will transmit the information to its susceptible neighbors. A node in the recovered state is "idle" and does not transmit any information. A unique feature, which makes our model distinct from the classic SAR model, is that there are two distinct ways for an adopted node to transit information to a susceptible neighbor, depending on whether the underlying social contact is close or ordinary. Empirical evidence [25-27] suggests that the transmission associated with a close contact contributes to reinforcement, but that with an ordinary contact does not. For a close contact, there is a memory effect in that the number $m$ of times that a susceptible node receives the information from the adopted neighbors is stored and used to determine the probability that the node actually adopts the behavior [13], 
making the underlying dynamics non-Markovian. However, for an ordinary contact, a susceptible individual would accept the behavior from any adopted neighbor with probability $p$. We subsequently extend our model to empirical networks.

The dynamical process of social contagion can be described, as follows. Initially, $E_{c}=E \mu$ edges are randomly selected as close contacts, associated with which is reinforcement. $\mu$ denotes the probability that an edge is assigned as a closed contact. The remaining edges represent ordinary contacts. A contagion is initiated within a single adopted cluster of size $N \rho_{0}$, while the remaining nodes are in the susceptible state. The spreading process starts and proceeds according to the SAR model, and the nodal dynamical states evolve using the synchronous updating rule. Specifically, an individual $(i)$ who has adopted the behavior attempts to transmit the behavioral information to its susceptible neighbors $(j)$. For a close social contact, the transmission from the adopted end to the susceptible end occurs with probability $q$, and $j$ will successfully adopt the message or behavior only if it has received the information at least $T$ times, where $T$ is the adoption threshold. In this case, there is reinforcement. For an ordinary contact, $j$ becomes adopted with probability $p$, or the transmission rate, without contributing to the accumulated times $m$. The spreading process is repeated until all adopted individuals become extinct in the network and the dynamics have reached a stable steady state.

For simplicity, we assume in our study that the recovery probability is $r=1$, i.e., an individual who adopts the behavior at time $t$ will transmit the behavior to all its susceptible neighbors at time $t+1$, after which it will lose interest in the behavior and will not transmit the message or behavior again. In all cases, we set $q=1.0$.

\section{THEORY}

We present a theoretical analysis to elucidate the roles of close and ordinary contacts in social contagion dynamics based on the edge-based compartmental theory [28-31]. In our model, a node $u$ in a cavity state cannot transmit the behavioral information to its neighbors but can receive it from others [32]. The dynamical correlation among the states of the neighbors is characterized by the two types of the contacts. If node $u$ with degree $k_{u}$ is susceptible at time $t$, then it does not belong to the cluster of initial seed of contagion and it receives the information less than $T$ times from close contacts. We write $k_{u}=k_{u}^{C}+k_{u}^{O}$, where $k_{u}^{C}$ and $k_{u}^{O}$ are the numbers of close and ordinary contacts of $u$, respectively. Let $\theta_{X}(t)(X \in\{C, O\})$ be the probability that, up to time $t$, the message or behavioral information has not been successfully transmitted from one end of one close or ordinary contact of $v$ to the other end $u$. Combining the two conditions, we obtain the probability that node $u$ is in the susceptible state as

$$
\begin{aligned}
s(k, t)= & \left(1-\rho_{0}\right) \sum_{k_{C}=0}^{k}\left(\begin{array}{c}
k \\
k_{C}
\end{array}\right) \mu^{k}(1.0-\mu)^{k-k_{C}} \\
& \times \sum_{m_{C}=0}^{T-1} \phi_{m_{C}}^{C}\left(k_{C}, t\right) \theta_{O}^{k-K_{C}}
\end{aligned}
$$

where $\phi_{m_{C}}^{C}$ is the probability that node $u$ has received $m_{C}$ pieces of information from close contacts by time $t$. The term $\sum_{k_{C}=0}^{k}\left(\begin{array}{l}k \\ k_{C}\end{array}\right) \mu^{k}(1.0-\mu)^{k-K_{C}}$ represents the probability that node $u$ has $k_{C}$ edges corresponding to close contacts. The detailed expression of $\phi_{m_{X}}^{X}$ is given by

$$
\phi_{m_{X}}^{X}\left(k_{X}, t\right)=\left(\begin{array}{c}
k_{X} \\
m_{X}
\end{array}\right) \theta_{X}^{k_{X}-m_{X}}\left(1-\theta_{X}\right)^{m_{X}} .
$$

The fraction of susceptible nodes is

$$
S(t)=\sum_{k} p(k) s(k, t) .
$$

We wish to obtain the expression of $\theta_{X}(t)$ according to its definition of $\theta_{X}(t)$. In our model, an edge of a susceptible individual $u$ can connect to a susceptible, an adopted or a recovered neighbor $v$. Accordingly, $\theta_{X}(t)$ consists of three parts:

$$
\theta_{X}(t)=\xi_{S}^{X}(t)+\xi_{A}^{X}(t)+\xi_{R}^{X}(t),
$$

where $\xi_{S}^{X}(t), \xi_{A}^{X}(t)$, and $\xi_{R}^{X}(t)$ are the probabilities that the neighbor is in the susceptible, adopted, and recovered state, respectively, and the information or message has not been successfully transmitted to $u$ up to time $t$.

If $v$ is in the susceptible state, then it cannot transmit the behavioral information or message to $u$. Moreover, because node $u$ is in the cavity state, node $v$ can only obtain the information or message from other neighbors excluding $u$. Up to time $t$, node $v$ with degree $k_{v}=k_{C}+k_{O}$ can obtain $m_{x}$ pieces of information from contacts of type $X$ with the probability

$$
\tau_{m_{X}}^{X}\left(k_{X}, t\right)=\left(\begin{array}{c}
k_{X}-1 \\
m_{X}
\end{array}\right) \theta_{X}^{k_{X}-m_{X}-1}\left(1-\theta_{X}\right)^{m_{X}} .
$$

Node $v$ will remain in the susceptible state if it has not received more than $T$ pieces of message or information through close contacts, neither has it received any message from any ordinary contact. There are two cases because the contact between $v$ and $u$ is either close or ordinary. Combining Eqs. (2) and (5), we obtain the probability that node $v$ is in the susceptible state in each case as

$$
\begin{aligned}
& \Theta_{C}(k, t)=\sum_{k_{C}=0}^{k}\left(\begin{array}{c}
k \\
k_{C}
\end{array}\right) \mu^{k}(1.0-\mu)^{k-k_{C}} \sum_{m_{C}=0}^{T-1} \tau_{m_{C}}^{C}\left(k_{C}, t\right) \theta_{O}^{k-k_{C}}, \\
& \Theta_{O}(k, t)=\sum_{k_{O}=0}^{k}\left(\begin{array}{c}
k \\
k_{O}
\end{array}\right) \mu^{k-k_{O}}(1.0-\mu)^{k_{O}} \sum_{m_{C}=0}^{T-1} \theta_{O}^{k_{O}-1} \phi_{m_{C}}^{C}\left(k_{C}, t\right) .
\end{aligned}
$$

From the degree distribution $p(k)$, we obtain the probability that the edge of type $X$ is connected to a susceptible neighbor as

$$
\xi_{S}^{X}(t)=\left(1-\rho_{0}\right) \frac{\sum_{k} k p(k) \Theta_{X}(k, t)}{\langle k\rangle},
$$

where $k p(k) /\langle k\rangle$ is the probability that an edge is connected to a neighbor of degree $k$ in an uncorrelated network. 
If $v$ is an adopted node, then it would transmit the information or message to a susceptible neighbor through an edge with probability $\lambda_{X} \in\{p, q\}$, leading to a decrease in $\theta_{X}(t)$. We thus have

$$
\frac{d \theta_{X}}{d t}=-\lambda_{X} \xi_{A}^{X}
$$

Otherwise, $v$ fails to transmit the information or message to neighbor and becomes recovered, $\xi_{R}^{X}$ will increase. This means that the increment $d \xi_{R}^{X}$ must satisfy two conditions: (a) the adopted neighbor has not transmitted the behavioral information or message to neighbors, which occurs with the probability $1-\lambda_{X}$, and (b) the adopted neighbor recovers with probability $\gamma$. We thus have

$$
\frac{d \xi_{R}^{X}}{d t}=\gamma\left(1-\lambda_{X}\right) \xi_{A}^{X}
$$

From Eqs. (9) and (10) as well as the initial conditions $\theta_{X}(0)=1$ and $\xi_{R}^{X}(0)=0$, we obtain

$$
\xi_{R}^{X}(t)=\gamma\left[1-\theta_{X}(t)\right]\left(1-\lambda_{X}\right) / \lambda_{X}
$$

Combining Eqs. (4), (8), (9), and (11), we obtain the following equation that governs the evolution of $\theta_{X}(t)$ :

$$
\begin{aligned}
\frac{d \theta_{X}(t)}{d t}= & \left(1-\rho_{0}\right) \lambda_{X} \frac{\sum_{k} k p(k) \Theta_{X}(k, t)}{\langle k\rangle} \\
& +\gamma\left[1-\theta_{X}(t)\right]\left(1-\lambda_{X}\right)-\lambda_{X} \theta_{X}(t) .
\end{aligned}
$$

Using the identity $S(t)+A(t)+R(t)=1$ and the fact that the rate $d A(t) / d t$ is equal to the rate at which $S(t)$ decreases, we have

$$
\begin{gathered}
\frac{d A(t)}{d t}=-\frac{d S(t)}{d t}-\gamma A(t), \\
\frac{d R(t)}{d t}=\gamma A(t),
\end{gathered}
$$

where $A(t)$ and $R(t)$ are the fractions of the adopted and recovered population at time $t$, respectively. Accordingly, $R(\infty)$ represents the final fraction of the recovered population after the system has reached a steady state that no longer has any adopted node. Equations (1)-(3) and (12)-(14) describe a full and general picture of the contagion dynamics.

The steady state fraction of nodes that have adopted the behavior can be obtained from Eq. (12) by taking the limit $t \rightarrow \infty$ as

$$
\begin{aligned}
\theta_{X}(\infty)= & \left(1-\rho_{0}\right) \frac{\sum_{k} k p(k) \Theta_{X}(k, \infty)}{\langle k\rangle} \\
& +\gamma\left[1-\theta_{X}(\infty)\right] \frac{1-\lambda_{X}}{\lambda_{X}} .
\end{aligned}
$$

It is now feasible to analyze the critical information transmission probability. In the presence of social reinforcement $(T>1)$, a vanishingly small number of seeds cannot trigger a global adoption. It is useful to consider a finite fraction of seeds: $\rho_{0}>0$. In this case, $\theta_{X}(\infty)=1$ is not a solution of Eq. (15). At the critical point of first-order phase transition, the condition

$$
\frac{\partial \theta_{C}\left[\theta_{C}(\infty), \theta_{O}(\infty)\right]}{\partial \theta_{O}(\infty)} \frac{\partial \theta_{O}\left[\theta_{C}(\infty), \theta_{O}(\infty)\right]}{\partial \theta_{C}(\infty)}=1
$$

is fulfilled [33], where $\theta_{X}\left[\theta_{C}(\infty), \theta_{O}(\infty)\right]$ is the right-hand side of Eq. (15).

\section{RESULTS}

\section{A. Erdös-Rényi (ER) networks}

We simulate the spreading dynamics on ER networks with $N_{r}=500$ independent realizations. A new random network with the same rewiring probability $p_{e}$ is built after every 25 independent realizations of the spreading dynamics. Unless otherwise specified, the simulation parameter values in most cases are $N=10^{4}, \rho_{0}=0.003, q=1.0$, and $\langle k\rangle=$ 10.0 (corresponding to $p_{e}=0.001$ ). The number of edges is thus $E=5 \times 10^{4}$. For small values of $\rho_{0}$, analytic prediction can be obtained to uncover the roles of the two distinct types of social contacts in contagion dynamics.

A fundamental feature of the spreading dynamics of social contagion is the emergence of phase transitions: as a system parameter varies through a critical point, an outbreak occurs. The transition can be continuous (second order) or discontinuous (first order). To gain insights into the role of close versus ordinary contacts in the characteristics of phase transition, we calculate the final fraction of the recovered population for different values of $T$ and $\mu$ numerically and theoretically, as shown in Fig. 1. In all cases, there is a phase transition. As the fraction of close contacts in the network is increased, there is a change in the nature of the phase transition from being continuous to discontinuous, where the abrupt change in the final recovered population at the transition point can be quite large for relatively large values of the fraction. The dynamical origin of the discontinuity can be attributed to the relative abundance of the close contacts that lay the ground for the occurrence of a drastic avalanche type of process, as suggested by the previous result that a sufficient number of close contacts with a relatively large transmission rate can trigger a massive outbreak of contagion [13,34]. For relatively higher values of the adoption threshold $T$, second order transitions are more likely, as illustrated in Fig. 1(c). The reason lies in the susceptible individuals in the critical state, i.e., the individuals who have been informed by their adopted neighbors through close contacts but have not adopted the message or information yet. For a large value of $T$, it is more difficult to make these individuals adopt the behavior, thereby requiring more transmission to trigger a massive adoption process. The contribution of reinforcement in this case is insignificant, leading to a smaller avalanche size [34,35]. Note the good agreement between numerical and theoretical results, which validates the edge-based compartmental approach.

Figure 1 also shows that close contacts of sufficient number tend to delay the outbreak of massive contagion, as the corresponding threshold values are larger than $T_{C}=\langle k\rangle /\left(\left\langle k^{2}\right\rangle-\right.$ $\langle k\rangle)=\langle k\rangle^{-1}$ as predicted by the bond-percolation theory for the conventional susceptible-infected-refractory (SIR) dynamics [36,37]. More close contacts are more effective at preventing a large-scale outbreak, regardless of the values of the transmission rate and adoption threshold $T$. This is quite surprising as previous work had concluded that dense contacts with social reinforcement would in general facilitate contagion [17]. 
(a)

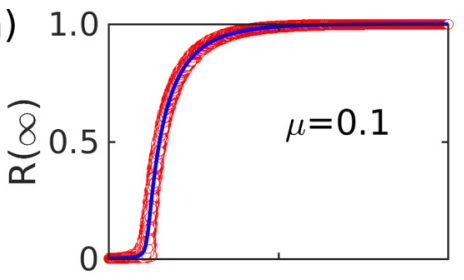

(b)

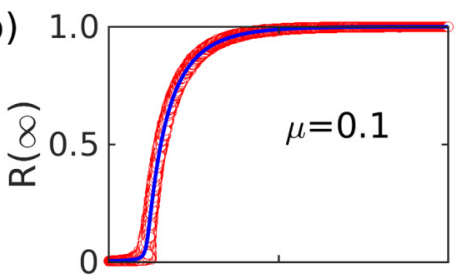

(c)

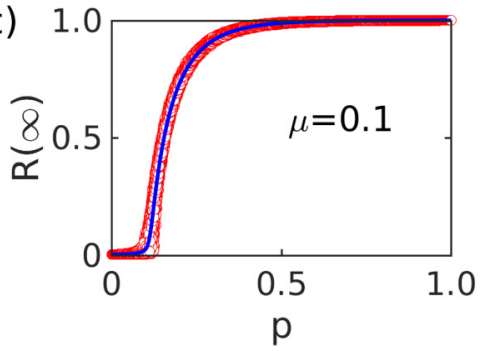

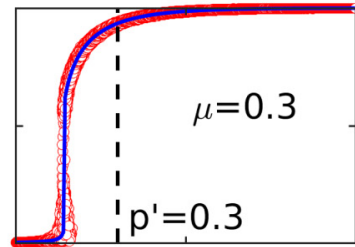
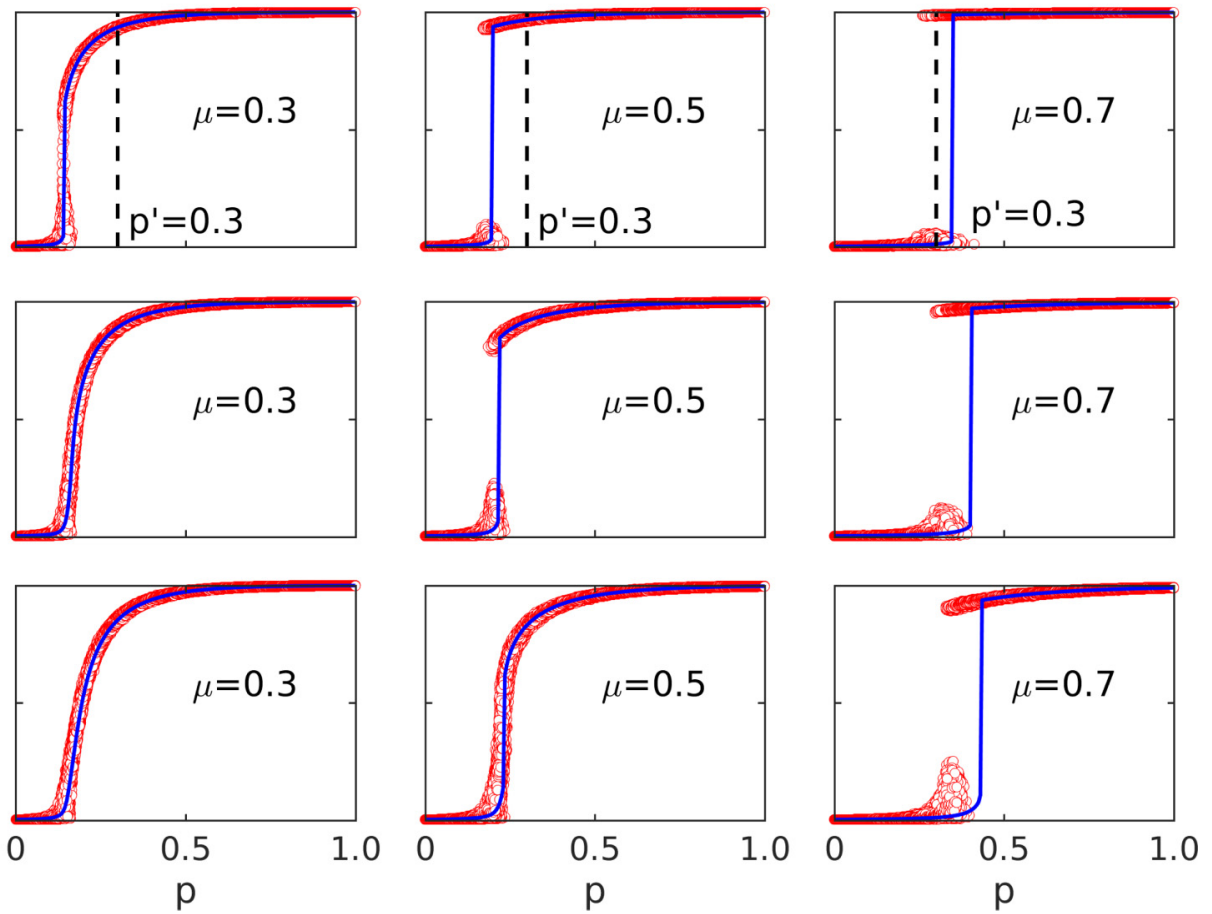

FIG. 1. Numerically obtained and theoretically predicted final fraction of the recovered population for different parameter values. Simulation results (red circles) and theoretical prediction of Eq. (12) (blue solid lines) for the final recovered population versus transmission rate $p$ for three different values of $T:$ (a) $T=3$, (b) $T=4$, and (c) $T=5$, where for each value of $T$, the results for four different values of $\mu$ are presented. Each data point is obtained from a single realization. The black vertical dashed lines indicating the position of $p^{\prime}=0.3$ are included in (a) for corresponding the results to the dynamical behaviors of the system in Fig. 2.

The counterintuitive phenomenon can be understood by estimating the contributions of the two types of distinct contacts to the spreading dynamics. Figure 2 shows the key statistical characterizing quantities for $p^{\prime}=0.3$ : the fraction of recovered population (the first column), the numbers of three types of transmission events (the second column), the distribution $P(L)$ of diffusion path lengths $L$ (the third column), and the frequency spectrum of the occurrence of ordinary transmission events in the various diffusion paths (the fourth column). More specifically, in our model, transmission events can be classified into three major categories: (a) transmission associated with ordinary contacts which occurs with probability $p$-ordinary transmission, (b) transmission along close contacts which is able to drive the individuals to successfully receive the information about the behavior for $m_{i}(t)<T-$ intermediate reinforced transmission, and (c) transmission associated close contacts which results in acceptance of the behavior when the condition $m_{i}(t)=T$ is met-successful reinforced transmission, which occurs with probability $q$. The numbers of the three types of transmission events are denoted as $n_{O}(t), n_{M}(t), n_{S}(t)$, respectively. The quantity $n_{D}(t)=n_{M}(t) /(T-1)-n_{S}(t)$ represents the required additional minimum number of transmission events to stimulate all the remaining susceptible individuals in the critical state, after stimulation from successful reinforced transmission. About the statistical distribution $P(L)$, we note that a diffusion path of length $L$ is a combination of $L_{S}$ successfully reinforced transmission events and $L_{O}$ ordinary transmission events: $L=L_{S}+L_{O}$. With respect to the frequencies of occurrence of the ordinary transmission events along various diffusion paths, each color square at the position $\left(L^{\prime}, L_{O}^{\prime}\right)$ of the spectrum represents the probability that there are $L_{O}^{\prime}$ ordinary transmission events along the diffusion path of length $L^{\prime}$, where the nonempty squares below the diagonal lines indicate that ordinary transmission events are engaged in propagating the contagion forward together with the successful reinforced transmission events.

Figure 2 illustrates a key feature of the system: When the number of ordinary transmission events approximately matches $n_{D}(t)$ during the spreading process, i.e., $n_{O}(t) \approx$ $n_{D}(t)$, the system evolves into the state of maximum contagion. For a relatively small value of $\mu$ [Fig. 2(a)], close contacts are insufficient so that the amount of susceptible individuals in critical state are less than ordinary transmissions ought to stimulate. For a larger value of $\mu$, ordinary transmission events are rare and the two types of reinforced transmission events decrease in number even for a fixed value of the transmission rate $p$. The third column of Fig. 2 indicates the existence of an appreciable number of diffusion paths of intermediate and long length (e.g., $L \geqslant 7$ ) for the case where contagion is prevalent [Figs. 2(a) and 2(b)], and that short paths dominate when contagion is prohibited [Fig. 2(c)]. The behavior of the recovered population size suggests that long diffusion paths favor contagion when the value of the transmission rate and the fraction of ordinary contacts are proper to enable the dynamics.

Which elements contribute to the long paths and how do they facilitate contagion? The fourth columns of Fig. 2 provide a partial answer. For the cases shown, short paths $(L \leqslant 5)$ are mostly due to ordinary transmission events, while 

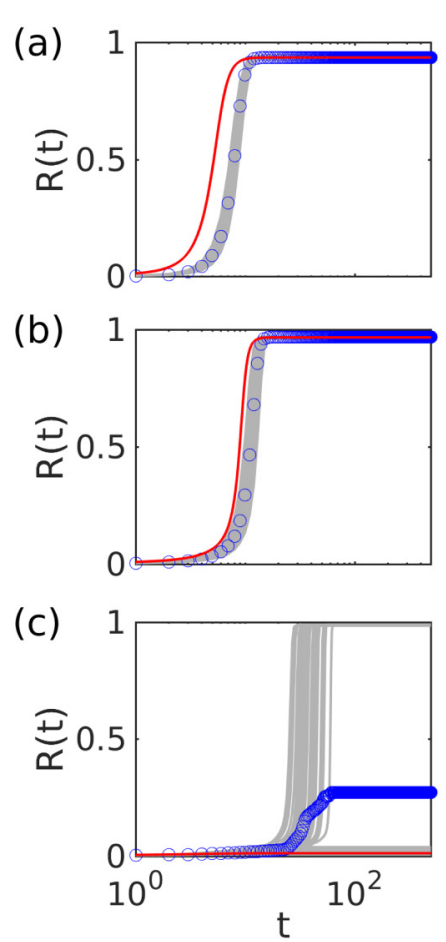
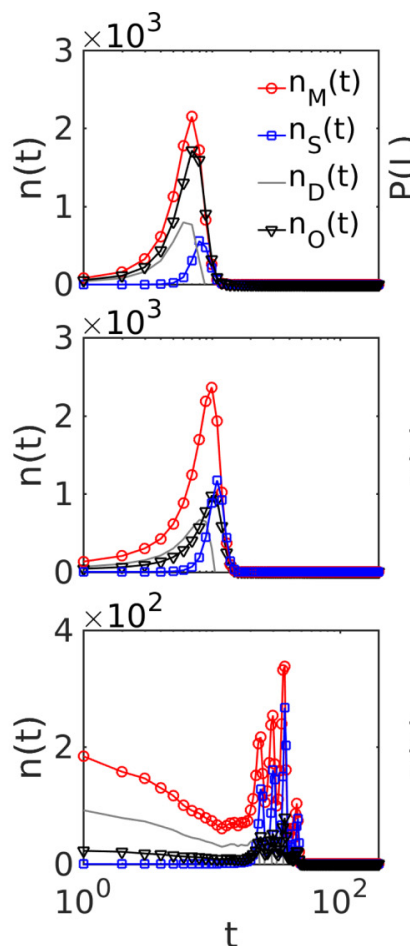
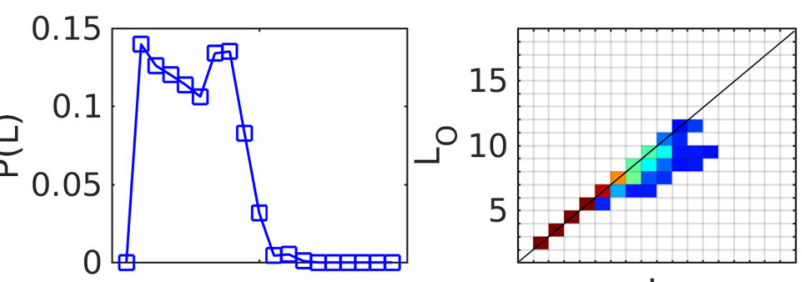

L
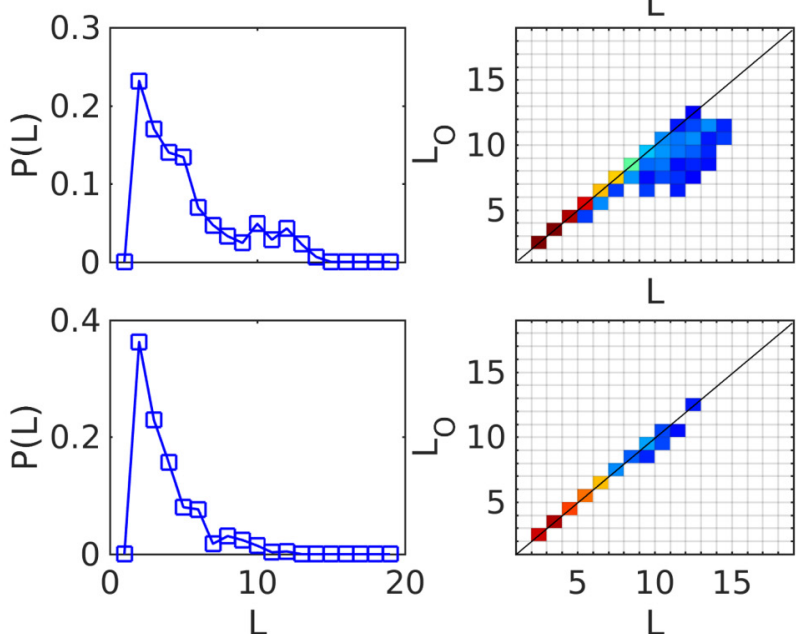

$\mathrm{L}$

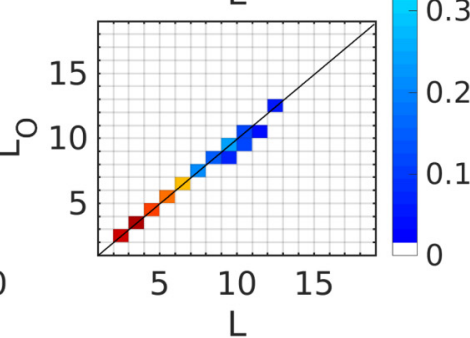

FIG. 2. Understanding the effects of ordinary contacts on spreading dynamics. Four key statistical characterizing quantities are shown for $p^{\prime}=0.3, T=3$, and $q=1.0$ : the fraction of recovered population (the first column), the numbers of three types of transmission events (the second column), the distribution $P(L)$ of diffusion path lengths $L$ (the third column), and the frequency spectrum of the occurrence of the ordinary transmission events in the various diffusion paths (the fourth column). Three different values of the parameter $\mu$ are used: (a) $\mu=0.3$, (b) $\mu=0.5$, and (c) $\mu=0.7$. The red solid lines in the first column are the analytical predictions, the blue circles represent the fractions of the recovered populations obtained by averaging $N_{r}$ independent realizations, and each light gray solid curve in each panel in the left column corresponds to one independent realization. The position of $p^{\prime}=0.3$ has also been specified in Fig. 1.

ordinary and successful reinforced transmission events have different contributions to longer diffusion paths for different values of $\mu$. In the fourth column of Figs. 2(a) and 2(b), the nonempty squares below the diagonal lines have a relatively wide distribution, indicating that ordinary transmission tends to "cooperate" with successful reinforced transmission to propagate the contagion forward together, enabling persistent transmission and generating long diffusion paths. Specifically, the more frequently ordinary transmission events are engaged in contagion propagation along longer paths (more nonempty squares below the diagonal lines in regions of large values of $L$ ), the population has more adopted individuals. That is, the existence of the long diffusion paths containing a substantial number of ordinary transmission events imply a long-time cooperative relationship between the two different types of transmission events. The extent of contagion is maximized when the number of susceptible individuals in the critical state induced by close contacts matches the number of ordinary transmission events as induced by ordinary contacts. There then exists an optimal fraction of close contacts to facilitate contagion to the maximum extent.

Note that, in the first column of Fig. 2(c), there is a discrepancy between the analytical and numerical results, especially near the critical point. This is due to the fluctuations as the system evolves from a local contagion state to a global prevalent state, as indicated by the spread in the gray curves from individual realizations.
The existence of an optimal fraction of close transmission events can be further illustrated numerically and analytically, as shown in Fig. 3, where the existence of the optimal fraction is more apparent for small values of $p$ and $T$. This means that the system can be harnessed to reach a maximum level of contagion without the need to change the transmission rate $p$. For a relatively large value of $p$ and a not too large value of $\mu$, ordinary contacts can be exploited to maximize the spreading in that it is more likely that the interplay between ordinary and successful reinforced transmission events will generate long diffusion paths. Note that the transitions with respect to varying $\mu$ are mostly discontinuous, especially in cases where there exists an interval of $\mu$ values with maximum spreading.

Figure 4 provides a visual picture of the results in Fig. 3, which further validates the physical picture for achieving maximum contagion in Fig. 2 through a match between the numbers of close and ordinary transmission events: $n_{m}(t) \gg$ $n_{e}(t)$ for $\mu_{2}=\mu_{o}=0.31$. Prevalence of the recovered population is not possible when close contacts dominate so there is a lack of cooperation with ordinary contacts, regardless of the occurrence of intermediate reinforced transmission [e.g., the second plot in Fig. 2(c)]. Overall, frequent cooperation between ordinary and successful reinforced transmission events along long diffusion paths is key to and plays a more important role in contagion prevalence rather than the mere existence of the long diffusion paths. Ordinary contacts are thus indispensable for successful social contagion. 
(a)

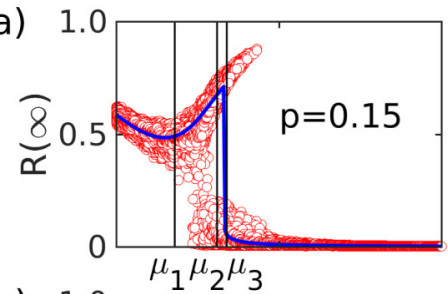

(b)

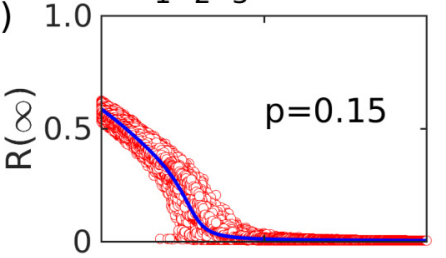

(c)

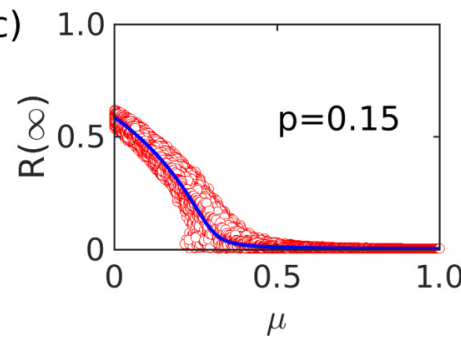

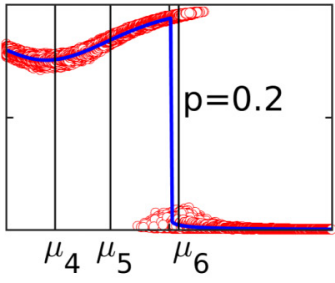
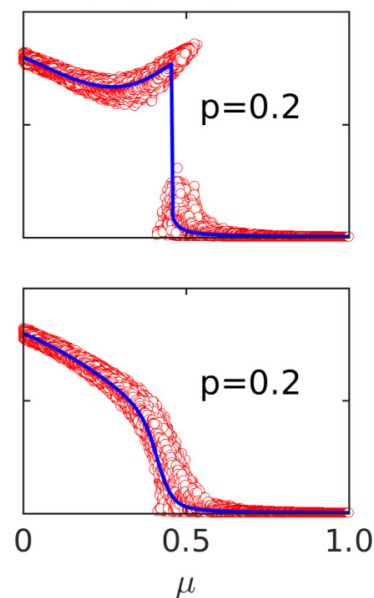
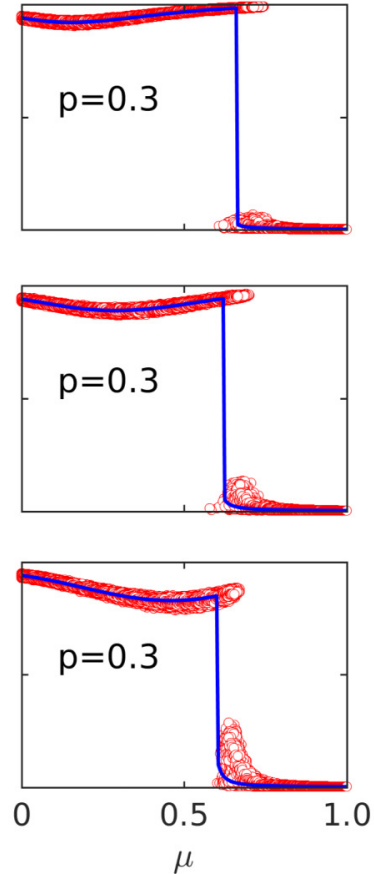
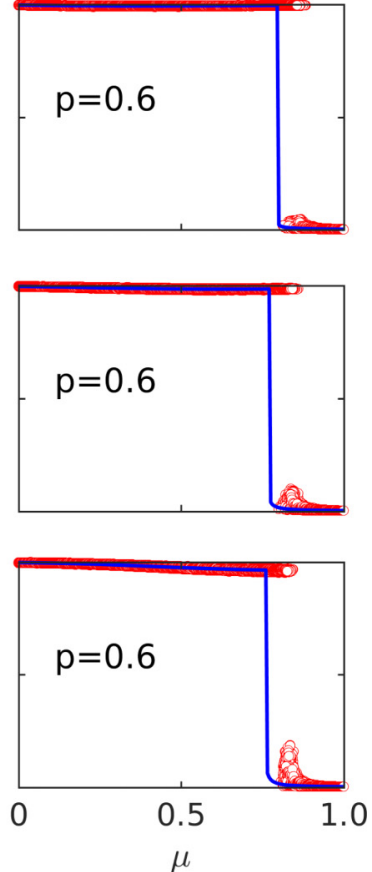

FIG. 3. Existence of an optimal fraction of close transmission events for global spreading. Shown is the final recovered population as a function of $\mu$ for three different values of $T$ for $q=1.0$ : (a) $T=3$, (b) $T=4$, and (c) $T=5$. For each value of $T$, simulation results (red circles) and analytical predictions (blue solid lines) are shown for four different values of $p$. Two groups of vertical solid lines $\left(\mu_{1}, \mu_{2}, \mu_{3}\right)$ and $\left(\mu_{4}, \mu_{5}, \mu_{6}\right)$ are indicated in (a), corresponding to Figs. 4 and 5, respectively.

Further support for requiring a nontrivial interplay between close and ordinary contacts in promoting large-scale spreading is provided in Fig. 5, where the colored square in the red circle reveals the cooperation between close and ordinary contacts along a long diffusion path of length $L=16$ that consists of 15 ordinary and one successfully reinforced
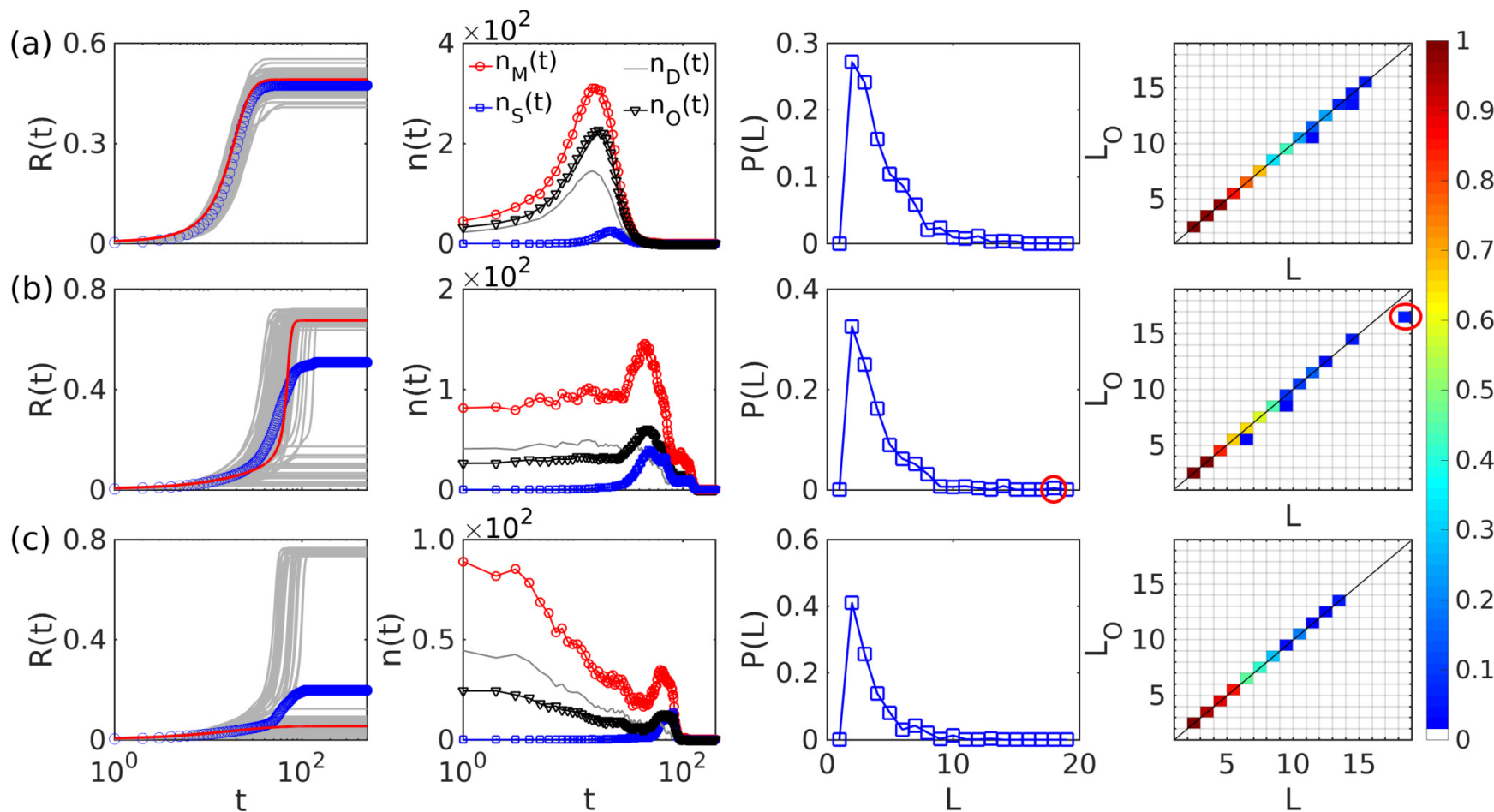

FIG. 4. Illustrations of the role of ordinary contacts in promoting spreading. Shown are the behaviors of four statistical characterizing quantities for different values of $\mu_{o}$ : (a) $\mu_{o}>\mu_{1}=0.18$, (b) $\mu_{o} \approx \mu_{2}=0.31$, and (c) $\mu_{o}<\mu_{3}=0.34$, the positions of which are also labeled in Fig. 3. Other parameter values are $p=0.15$ and $q=1.0$. 

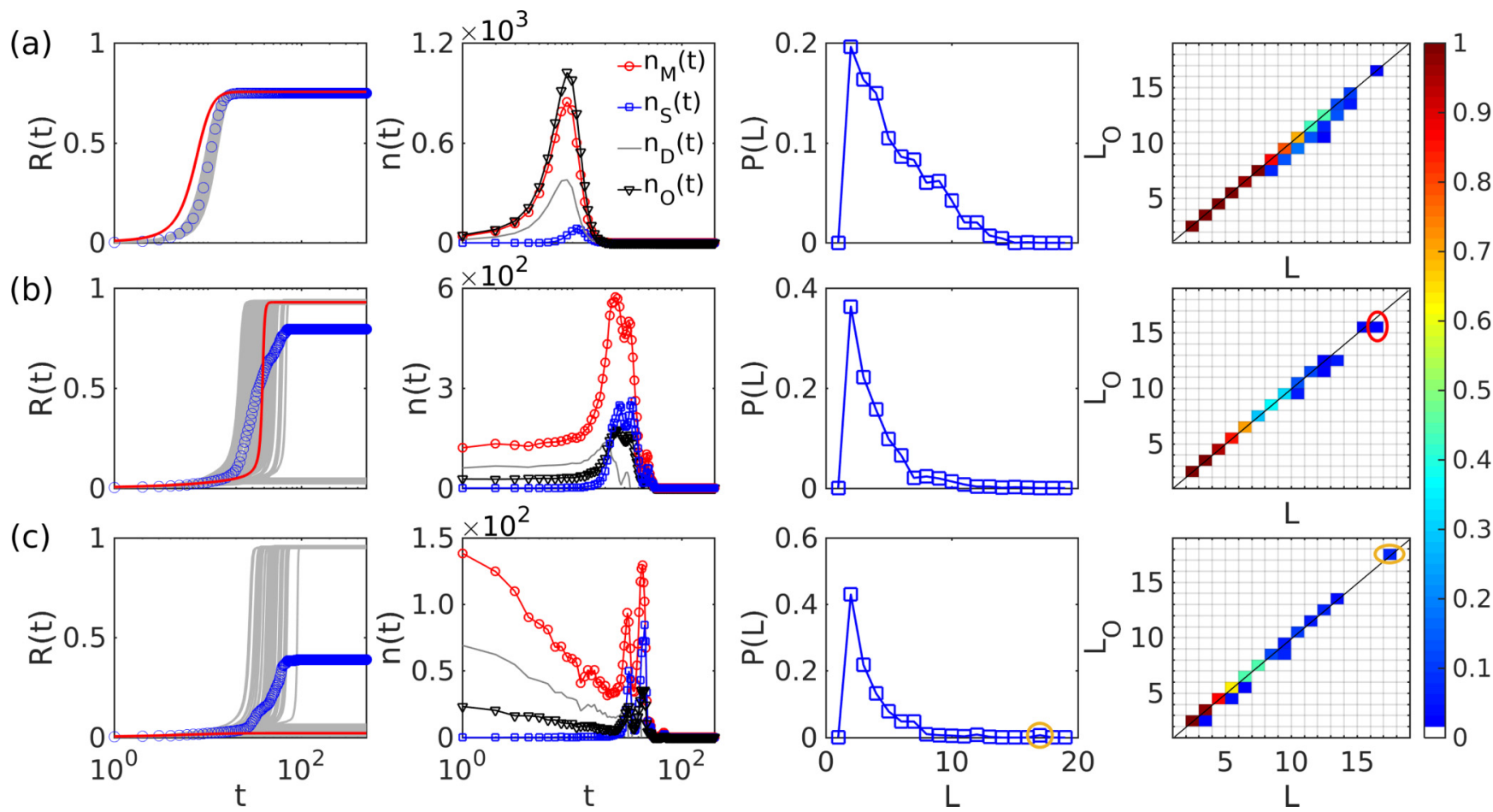

FIG. 5. Further illustrations of the role of ordinary contacts in promoting spreading. Shown are the behaviors of the four statistical characterizing quantities for (a) $\mu_{4}=0.15$, (b) $\mu_{5}=0.48$, and (c) $\mu_{6}=0.53$, corresponding to the three points of $\mu$ labeled in the second panel of Fig. 3(a). Other parameters are $p=0.2$ and $q=1.0$.

transmission events. Note that, in this case, longer diffusion paths exist, e.g., a path of length $L=17$, which consist of only one type of transmission events for small contagion size (the third and fourth plots in Fig. 5(c) - the symbols in the brown circles). However, these paths do not contribute to global spreading. The main reason for the discrepancies between analytical predictions and simulated results illustrated in the first columns of Figs. 4(b), 4(c) and 5(b), 5(c) is again the increasing fluctuations of the system near the outbreak threshold.

The phase transition scenarios of the system can be graphically understood through a bifurcation analysis of Eq. (15), as shown in Fig. 6. There are three distinct scenarios: continuous transition [Fig. 6(a)], transition at the triple point [Fig. 6(b)], and discontinuous transitions [Fig. 6(c)]. For the continuous transition, the graphical solution (the crossing point between the two relevant curves) moves gradually away from the position of $\theta_{s}=1$ and $\theta_{n}=1$ to that of $\theta_{s}<1$ and $\theta_{n}<1$. The crossing point begins to leave the top right corner of the plot at the critical point $p=p_{C}$. Associated with the triple point transition, although there exists one crossing point, two curves are close to each other near the crossing point that tends to move abruptly, which is indicative of a discontinuous transition. Note that the triple point can be estimated with the aid of pattern features of the solution lines, as illustrated in Fig. 6(b). For the discontinuous transitions, a solution for which the crossing points decrease from three (corresponding to contagion decay) to two (corresponding to contagion outbreak) until only one (contagion prevailing). Furthermore, Eq. (16) gives the tangent point of the two curves presented in the second subplot of Fig. 6(c). The crossing point (yellow solid circles) closest to the top right corner of the plot (i.e., $\theta_{s}=1$ and $\left.\theta_{n}=1\right)$ is physically meaningful, because the dynamics start from the initial conditions $\theta_{s} \approx 1$ and $\theta_{n} \approx 1$. The movement of the yellow points is caused by the shift of blue lines, indicating again the role played by the transmission events due to ordinary contacts in the phase transition.

Figures 7 and 8 provide a comprehensive picture in the parameter plane of $(\mu, p)$ of the spreading dynamics, where the former is numerically obtained while the latter is theoretical predictions. Note that the numerically identified critical boundaries and the analytically estimated triple point are included. Both figures represent evidence for the coexistence of continuous and discontinuous phase transitions separated by the triple point. More specifically, smooth color variations and dramatic differences in the color indicate continuous and discontinuous types of transition, respectively. There is an overall good agreement between the simulation results and the analytic predictions, although there is deviation between the two types of results in the region of large values of $\mu$. The discrepancies are somewhat expected, due to the numerical uncertainties in identifying the outbreak phase of the contagion through a finite number of statistically independent realizations. In general, the analytical predictions are more reliable, as evidenced by the better agreement with the numerical results for the limited parameter setting in which more extensive stochastic simulations are computationally feasible (e.g., the results in Figs. 1 and 3). The results in Figs. 7 and 8 indicate that the threshold and transition classes are predominately determined by the relative abundance of close contacts with respect to ordinary contacts in the underlying social network. Another feature is that the spreading dynamics 

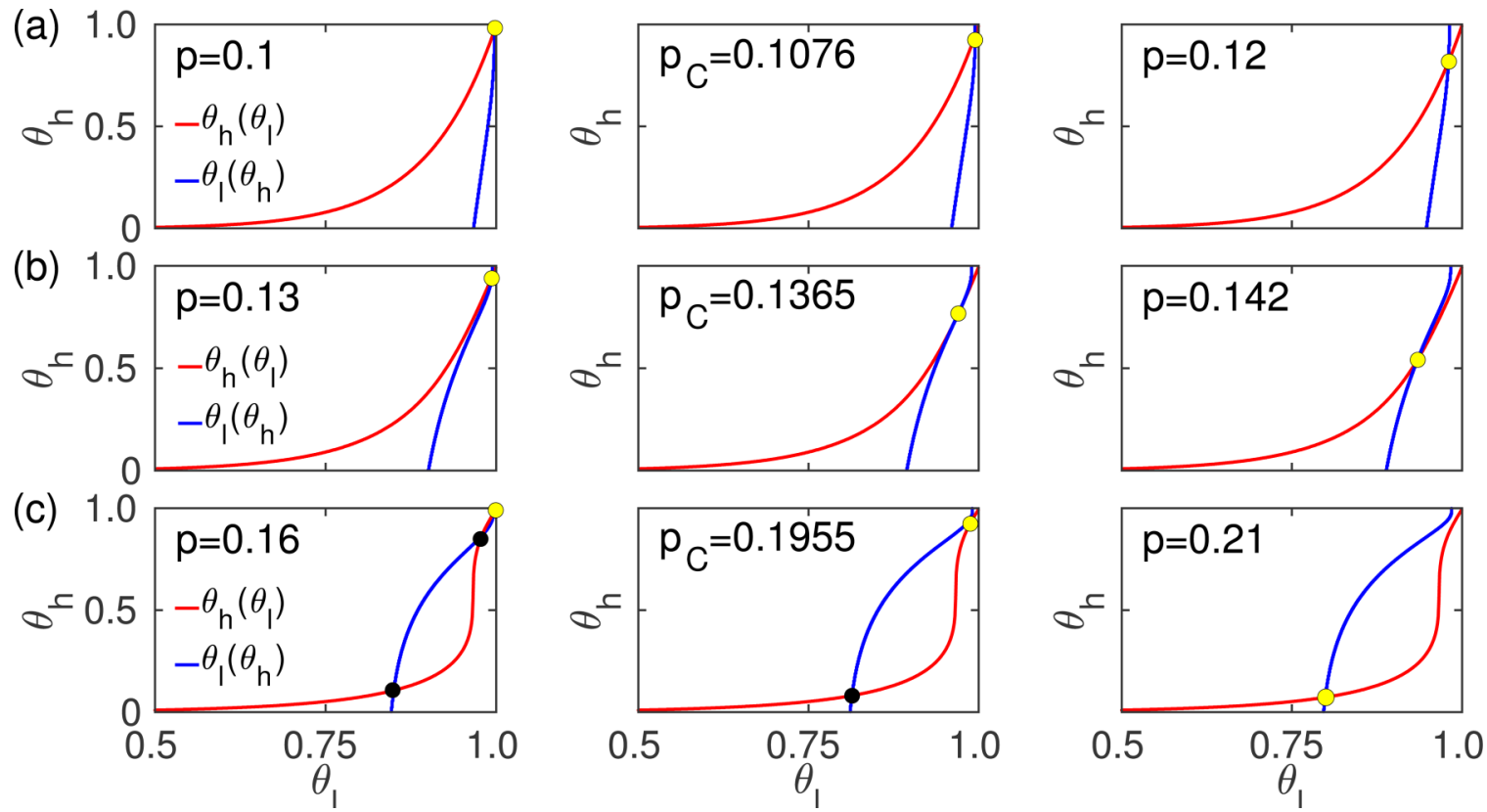

FIG. 6. Illustrations of graphical solutions of the bifurcation analysis for three cases: (a) continuous (second order) transition for $\mu=0.1$; (b) transition crossing the triple point for $\mu_{C}=0.245$; (c) discontinuous (first order) transition for $\mu=0.5$. Other parameters are $T=3$ and $q=1.0$.

in the presence of reinforcement $(T>1)$ is completely suppressed when the value of $\mu$ exceeds certain threshold whose value decreases with $T$, due to the lack of match between the two types of social contacts. Note that, in Figs. 7 and 8, the boundaries of continuous transitions are longer for higher values of $T$, a result that is consistent with those in Fig. 1.

Figure 9 shows, for ER random networks, the final stationary distributions of the recovered population for three values of the average degree $\langle k\rangle$ (or equivalently $p_{e}$ ). We observe that a relatively high average degree tends to facilitate the expansion of the contagion. Especially, a large average degree means that the adopted individuals will have more chances to transmit the contagion to their connected susceptible neighbors, resulting in a larger contagion size and a smaller outbreak threshold. At the same time, for a fixed value of $\mu$, the individuals will have more close contacts, so that more susceptible individuals can reach the critical state, leading to relatively more massive adoption within a short time and
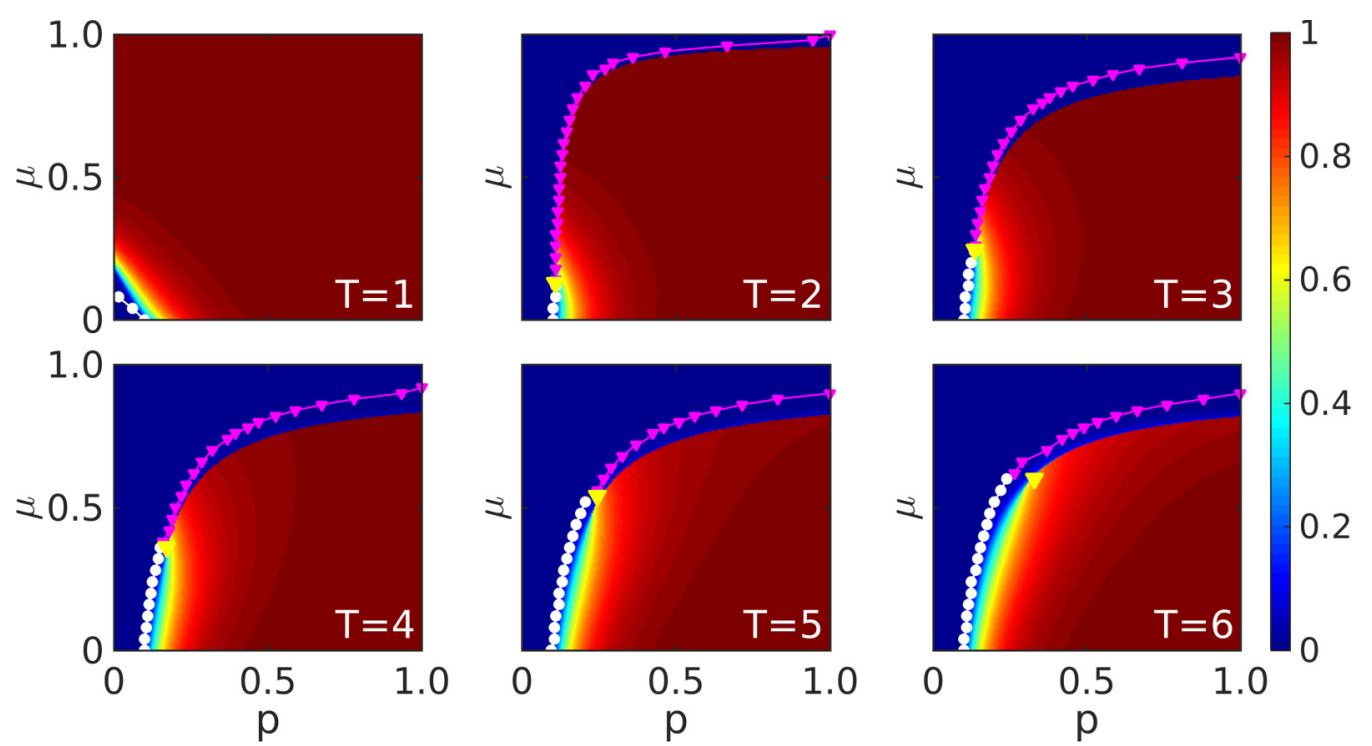

FIG. 7. Numerically obtained final stationary distributions of the recovered population. Shown are the distributions versus $p$ and $\mu$ for different values of $T$ and $q=1.0$. The large yellow inverted triangle represents the theoretically estimated triple point, while other markers denote the numerically estimated phase boundaries in terms of the relative variance $v(\infty)$. The white circles (pink inverted triangles) represent the boundaries across which continuous (discontinuous) transitions occur. 

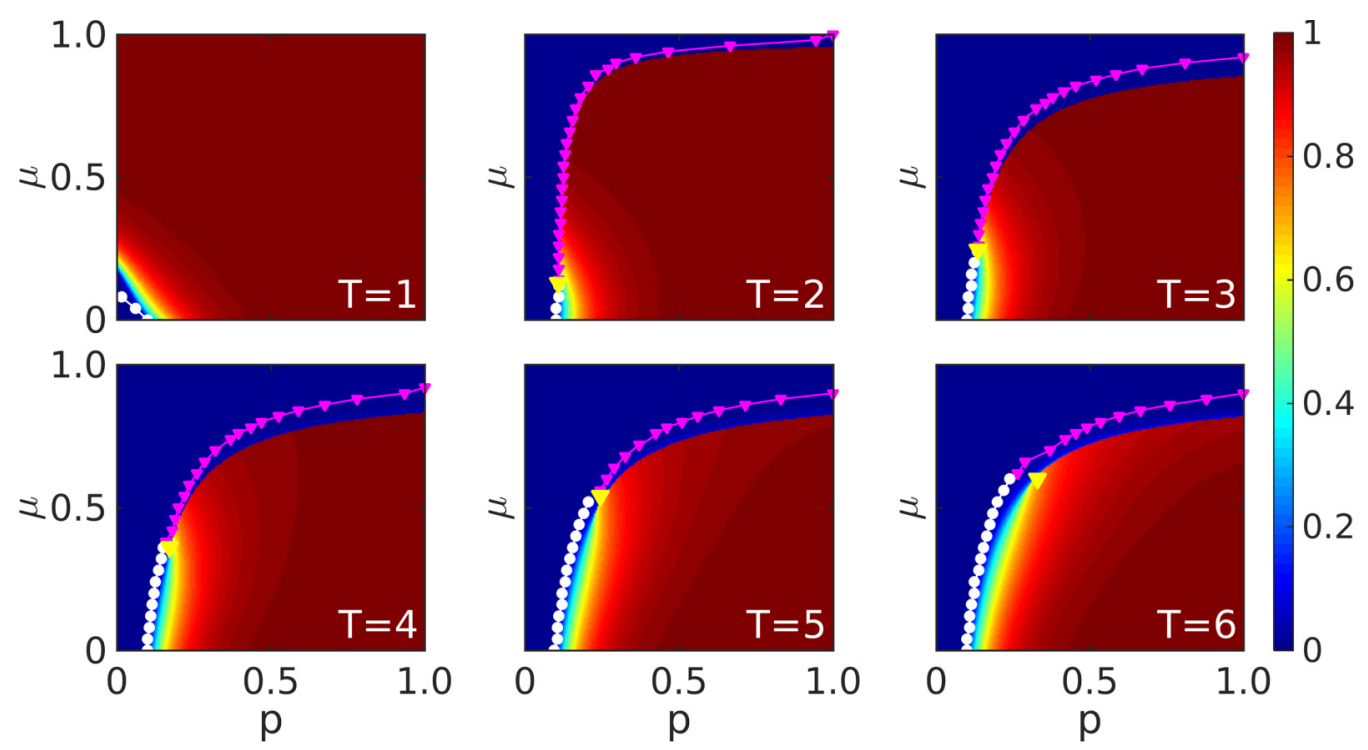

FIG. 8. Theoretically predicted final stationary distributions of recovered population. Legends are the same as those in Fig. 7. There is a good agreement between the numerical results in Fig. 7 and the theoretical predictions here.

an avalanchelike outbreak through subsequent transmission. Thus, as the value of the average degree is increased, more discontinuous transitions occur at the critical boundaries.

\section{B. Contagion dynamics on empirical networks}

We test our spreading model using two empirical networks: an online contact network (pretty good privacy (PGP) [38]) and the social network Brightkite [39]. Both networks have a heterogeneous degree distribution with a substantial amount of clustering, as shown in Fig. 10. In the simulations, the contagion starts from an adopted cluster of size $G(0)=30$.
Study of the empirical networks has led to phenomena that are not present in ER random networks, indicating the role of the network structure in the social contagion dynamics. Representative results are shown in Figs. 11 and 12. Because of the heterogeneous connections of the individuals in the network, the phase transition is continuous, regardless of the value of $\mu$, in agreement with the findings of the previous works on threshold based dynamics in heterogeneous networks $[13,16,40]$. In particular, in such cases, the number of susceptible individuals' being able to reach a critical value within a short time is not sufficient to lead to an avalanche-like outbreak. Another phenomenon is that, when the fraction
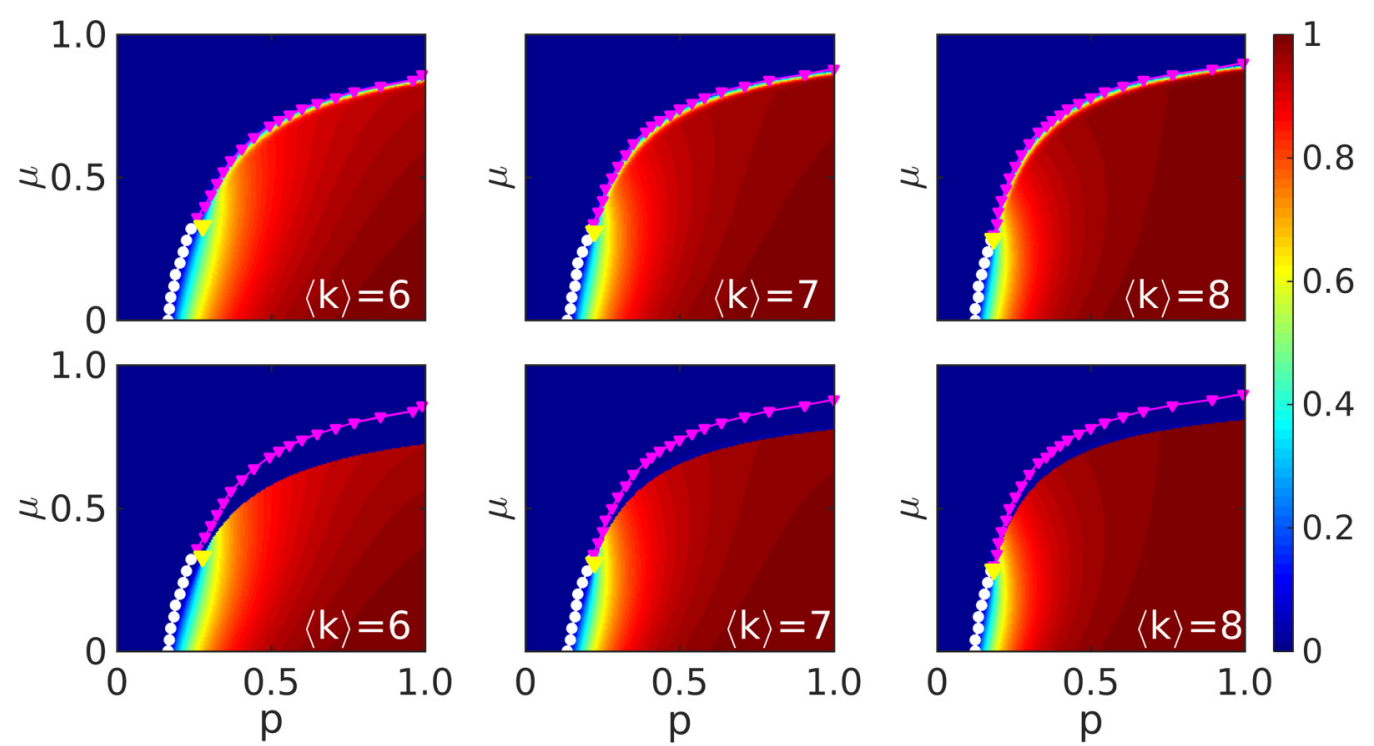

FIG. 9. Effect of average degree on the contagion dynamics. Shown are the color coded, numerically calculated (top panels) and theoretical predicted (bottom panels) final stationary distributions of recovered population for ER random networks in the parameter plane $(p, \mu)$. The values of the average degree tested are $\langle k\rangle=6$ (left column), $\langle k\rangle=7$ (middle column), and $\langle k\rangle=8$ (right column), with the corresponding values of $p_{e}$ being $p_{e}=0.0006, p_{e}=0.0007$, and $p_{e}=0.0008$, respectively. The main feature is that a relatively high average degree tends to facilitate contagion spreading. Other parameters are the same as those in Fig. 7. 

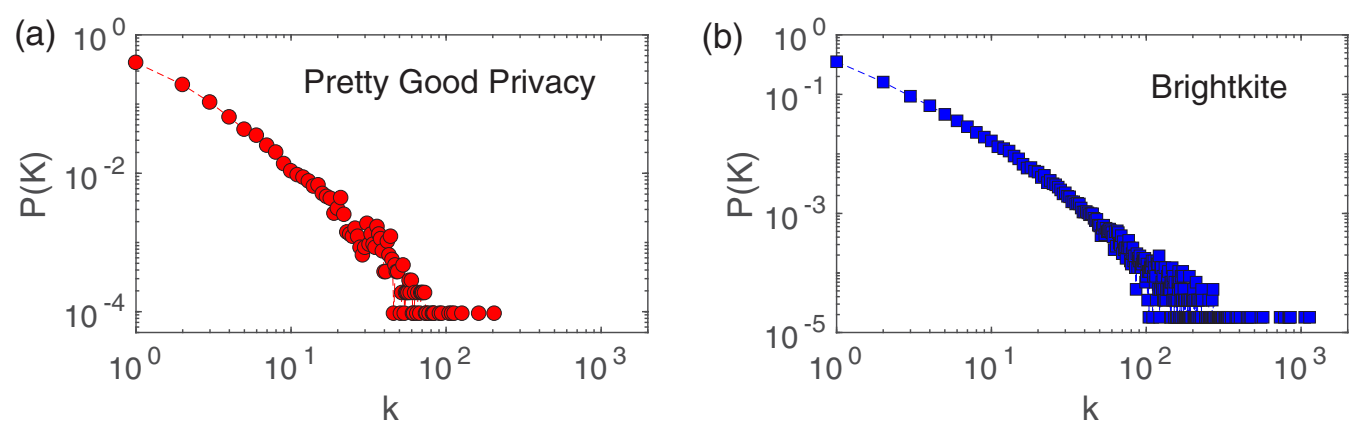

FIG. 10. Degree distributions of the two empirical networks. (a) The network pretty good privacy (PGP). The structural parameters are $N=10680,\langle k\rangle=4.554,\left\langle k^{2}\right\rangle=85.976$, maximum degree $k_{\max }=205$, degree-degree correlation $r=0.238$, and clustering coefficient $c=$ 0.378. (b) The network Brightkite. The structural parameters are $N=56739,\langle k\rangle=7.506,\left\langle k^{2}\right\rangle=480.61$, maximum degree $k_{\max }=1134$, degree-degree correlation $r=0.01$, and clustering coefficient $c=0.111$.

of closed contacts is large and the value of $T$ is relatively small, the threshold value is close to zero, suggesting that the outbreak of the contagion is largely driven by the hubs in the networks. However, the contagion size near the outbreak threshold is finite.

A heuristic mechanism leading to the phenomena in Figs. 11 and 12 can be described as follows. For a small value of $T$, there can be two different cases in terms of two parameter regions divided by a numerically estimated threshold $p_{h}$, where $p_{h}$ indicates the position at which the quantity $\Delta(p)$ begins to change from being positive to being negative. The results in Fig. 11 imply the following formula for determining the value of $\Delta(p)$ :

$$
\Delta(p)=\sum_{\mu}[R(p, \mu+\delta)-R(p, \mu)],
$$

where $\Delta(p)>0[\Delta(p)<0]$ means that the final contagion size increases (decreases) gradually with $\mu$. This argument relies on one condition: for a fixed value of $p$, the final fraction $R(p, \mu)$ of the recovered population should change monotonically with $\mu$, which has been verified numerically.

For $p<p_{h}$, abundant close contacts in the network can promote the contagion and lead to an outbreak, in contrast to ER random networks where this happens only for $T=1$. Because of the random allocation strategy of closed contacts among all connections, hubs have a natural advantage to possess a considerable number of close contacts. Evidence for this is presented in Figs. 13(a), which show the expected numbers of the close contacts of nodes in different degree classes with the probability of an edge to be a close contact being $\mu$. As a result, the hubs can readily enter the adopted state. For a large value of $\mu$, there is a higher probability for the contagion to be successfully transmitted among the hubs through close contacts, especially when the ordinary contacts have not been engaged in the transmission process (for small values of $p$ ). Evidence for this is presented in Figs. 13(b)-13(d). The hubs
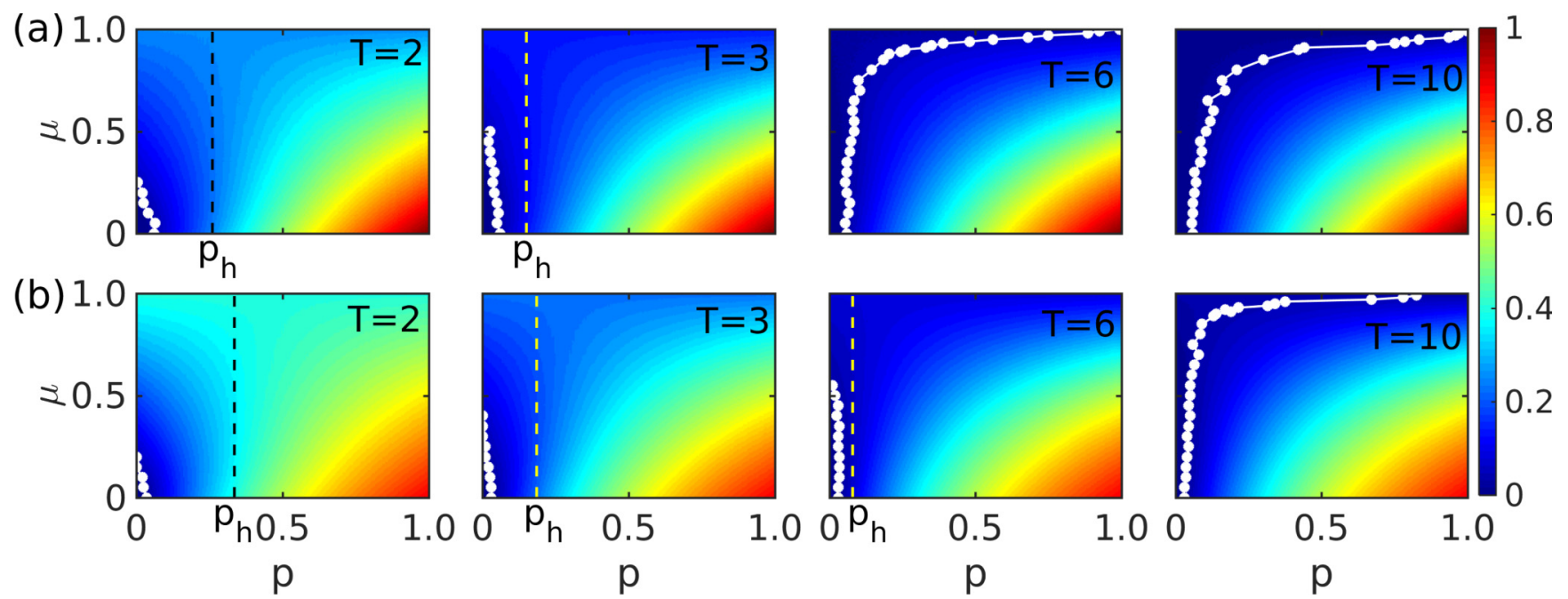

FIG. 11. Contagion dynamics and phase transition on the empirical network pretty good privacy (PGP). (a) Numerically obtained final stationary distributions of the recovered population. (b) The corresponding result from randomly-reconnected networks with the degree of each node unchanged (first-order null network model). In detail, a new first-order null network with the same degree sequence of PGP is built after every 25 independent realizations of the spreading dynamics. The solid white circles indicate the numerically estimated phase boundaries with respect to the relative variance $v(\infty)$. The dashed lines indicate the position of $p_{h}$, which are absent for $T>6$ in the top panels and $T=10$ in the bottom panels because closed contacts tend to block the contagion when the value of $T$ becomes large. 


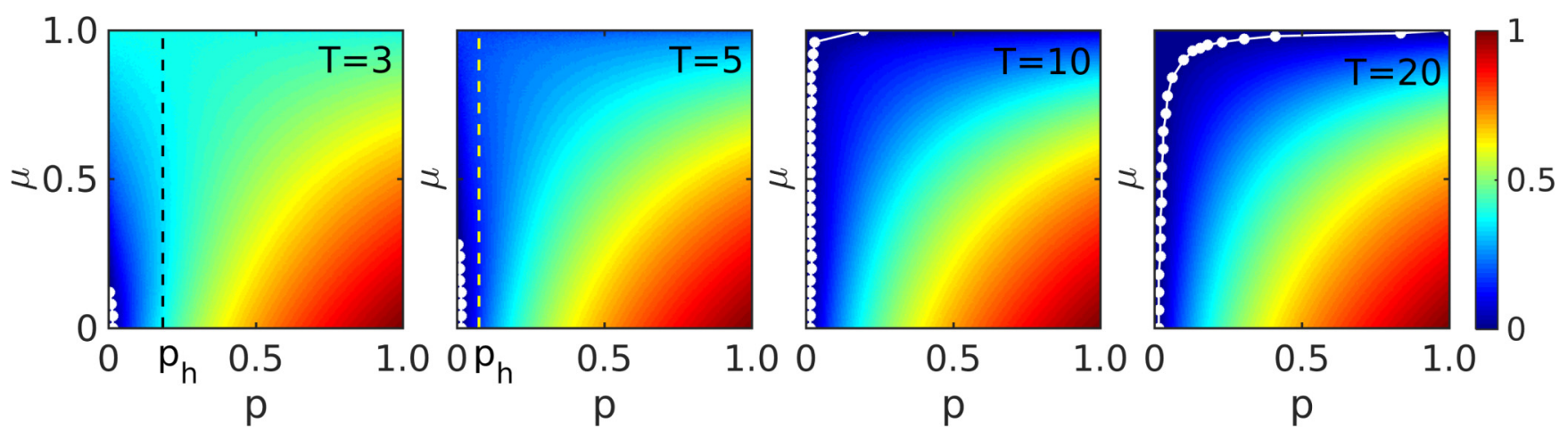

FIG. 12. Contagion dynamics and phase transition on the empirical network Brightkite. Legends are the same as in Fig. 11. Due to the weak degree correlation $(r=0.01)$ of this network, testing the first-order null network model is not essential.

can then facilitate the transmission, especially for networks with weak local clustering. Note that the closed contacts capable of successfully transmitting the contagion tend to locate near the hubs, especially for the empirical network with a high positive degree-degree correlation [Fig. 11(a)]. However, this localization effect is weakened in the first-order null network model of PGP, as the hubs have more chances to connect with nonhub nodes, making a larger adopted population possible, as shown in Fig. 11(b). Nonetheless, global contagion does not arise near the outbreak threshold because of the small value of $p$ - in this case contagion pathways of long distances from the hubs are less likely.

As the value of $p$ is increased toward $p_{h}$, more and more ordinary contacts begin to engage in the transmission process.
The contagion is again no longer restricted to local regions, and global contagion can occur through the transmission along the long-range ordinary contacts. By comparing with the results for ER networks, similar dynamics of the contagion but without discontinuous transitions for large $T$ can be observed. In the same way, sufficiently many close contacts in the network can inhibit global contagion. In such cases, in a network without close contacts, contagion can be maximized insofar as the value of the transmission rate $p$ is sufficiently large.

The similar dynamical behaviors in the PGP network and its first-order network model suggest that the contagion spreading depends more on the network heterogeneity than the degree-degree correlation. In fact, the correlation serves to
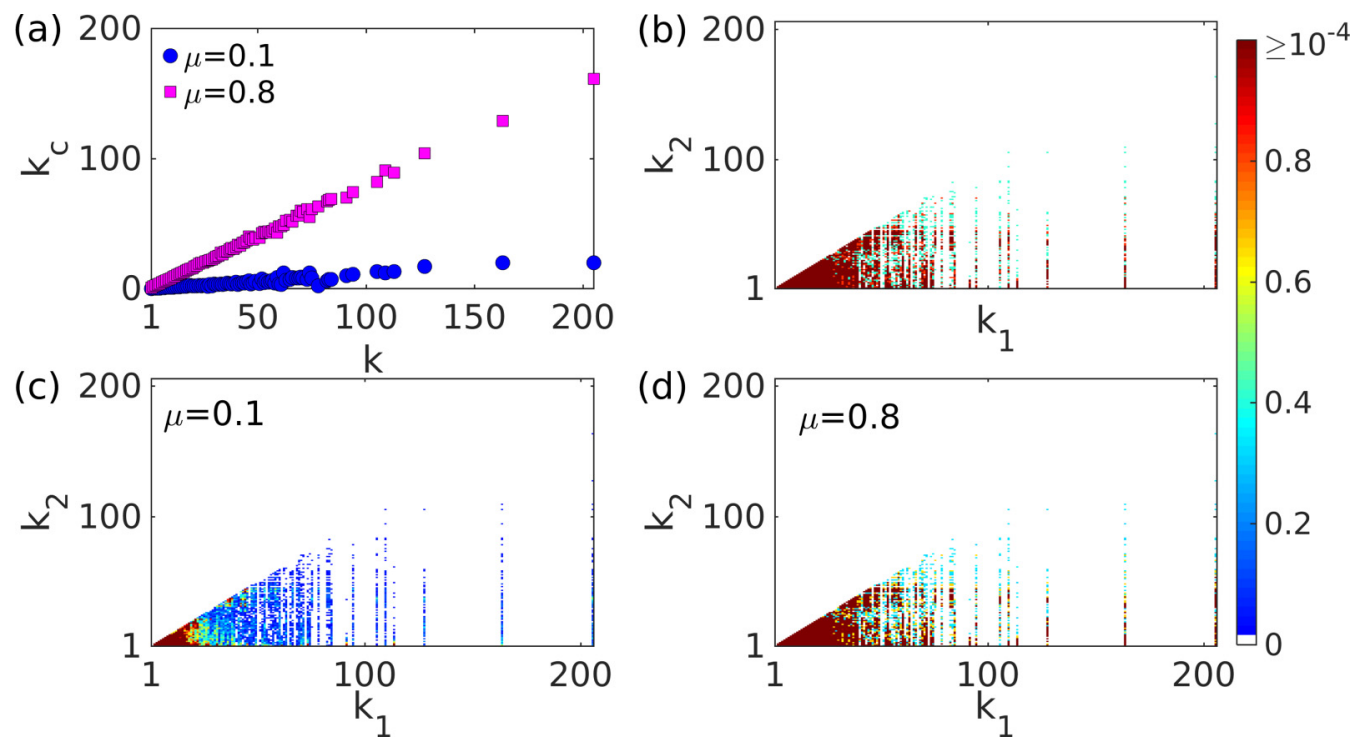

FIG. 13. Statistical behaviors of close contacts in the PGP network. Three key statistical characterizing quantities of close contacts are shown: (a) the expected number of close contacts that each node in different degree class can own for two different values of $\mu$, (b) the distribution of the edges between two nodes (node 1 and node 2, where $k_{1}$ and $k_{2}$ are the degrees of the two end nodes of an edge), normalized by the total number $E$ of edges in the network, and (c) the distribution of the close contacts between two nodes for $\mu=0.1$, normalized by $E$. (d) The distribution of the close contacts between two nodes for $\mu=0.8$. Because of the symmetry in the distribution, only half of the matrix is given in (b, c), where each color point indicates the value of $d\left(k_{1}, k_{2}\right)$ or $d\left(k_{2}, k_{1}\right)$, and $d\left(k_{1}, k_{2}\right)$ is the fraction of edges with two nodes of degree $k_{1}$ and $k_{2}$, respectively. The pink squares in (a) indicate that those nodes of high degree have considerable numbers of close contacts - a great advantage for transmission. The contagion spreads readily among these hubs due to the sufficient close contacts among them. The adopted areas are localized for small values of $p$. Similar phenomena are also found for the first-order null network model of PGP. 
(a)

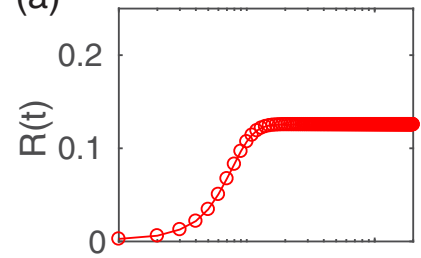

(b)

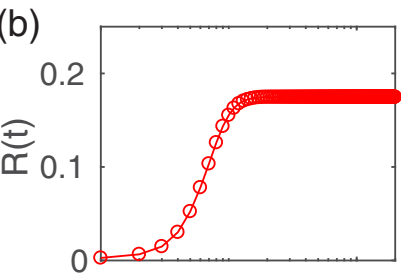

(c)

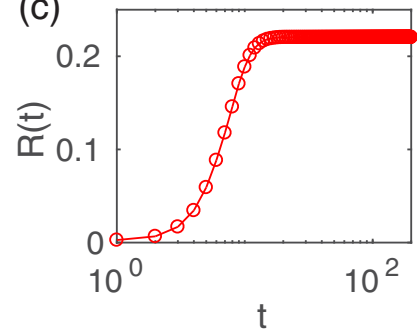

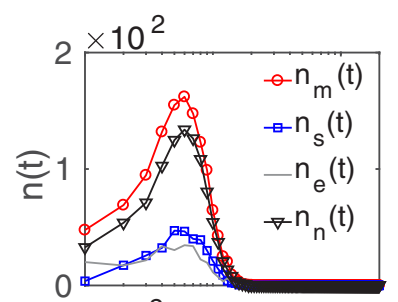
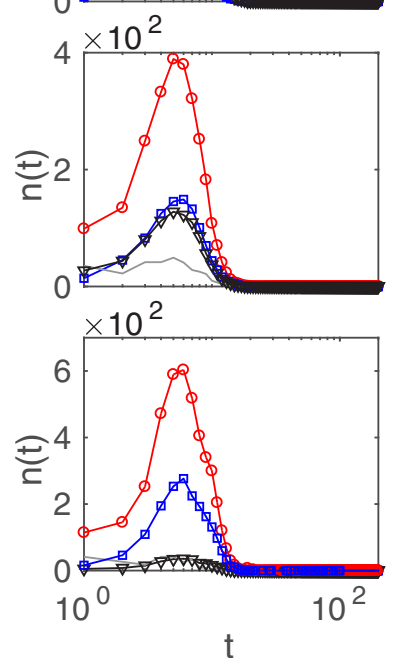
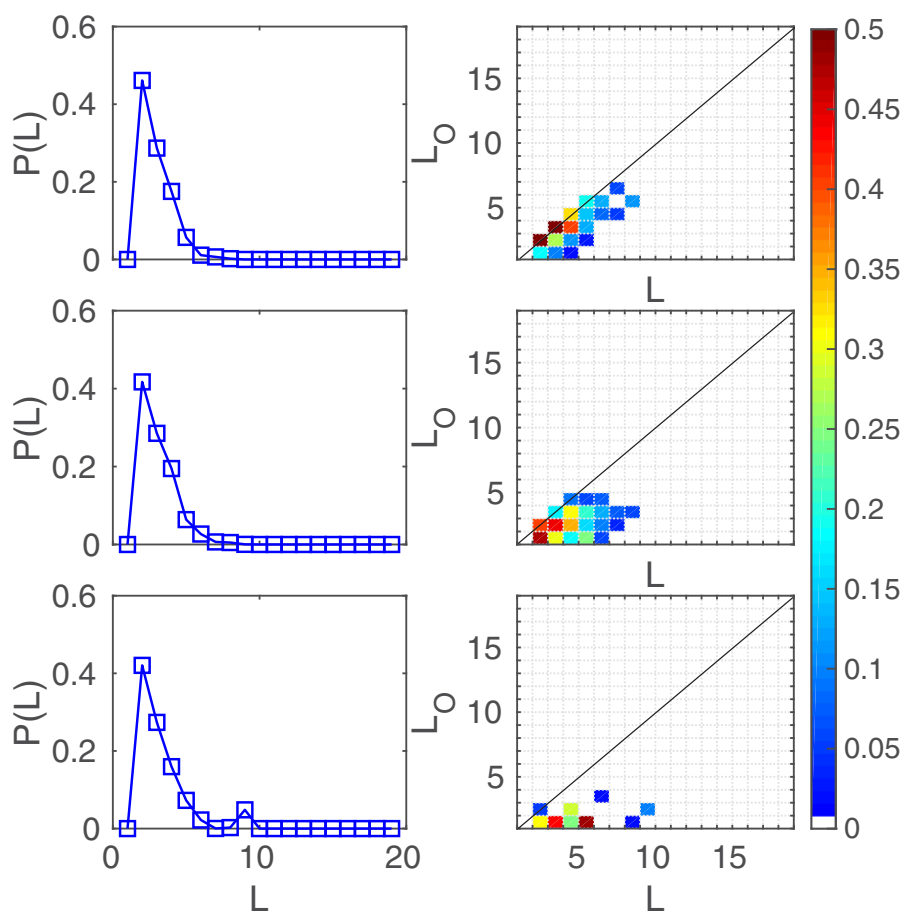

FIG. 14. The roles of two types of contacts in spreading dynamics in the PGP network. Shown are the behaviors of four statistical characterizing quantities for different values of $\mu$ : (a) $\mu=0.1$, (b) $\mu_{2}=0.4$, and (c) $\mu=0.8$, the positions of which are also labeled in Fig. 2. Other parameter values are $p_{h}>p=0.16, q=1.0$, and $T=2$.

delay the outbreak of the contagion, as evidenced by the larger outbreak thresholds in Figs. 11(a) and 12.

Two other phenomena in Figs. 11 and 12 are (1) the threshold $p_{h}$ disappears for large values of $T$ and (2) the parameter regions of global prevalence tend to shrink somewhat with $T$. The reason for the former is that a larger threshold value for successful adoption makes it harder for not only ordinary but also hub nodes to adopt the contagion through close contacts. Especially, hub nodes no longer have the advantage to take lead in promoting the spreading. Outbreak of the contagion is thus delayed or even completely suppressed by abundant close contacts in the network, as for ER networks. Instead, ordinary contacts play an increasingly pivotal role in dominating the contagion prevalence.

Surprisingly, the phenomenon that contagion is maximized when the roles of the two types of ties are comparable ceases to exist in the two empirical networks. Some indications are shown in Figs. 14 and 15, where the third columns of the both figures suggest the absence of diffusion paths of long length $(L>10)$ and the dominance of short paths. This is due to the lack of long-time cooperation between successful reinforced and ordinary transmission, as shown in the forth columns of Figs. 14 and 15. In particular, there is still cooperation between the two types of transmission events, but it is limited to short diffusion paths (see the third columns of Figs. 14 and 15 ), regardless of the value of the transmission rate $p$. As a result, an avalanche type of spreading process fails to persist at large scales. Overall, heterogeneous networks do not provide the structural condition for cooperation between closed and ordinary contacts along long paths.

\section{DISCUSSION}

To summarize, empirical evidence and understanding of social contagion suggest the indispensable roles played by ordinary and close contacts in the spreading dynamics, calling for a general model to take into account both types of social contacts. We have accomplished that in this paper. In addition, we have developed a theoretical approach to analyzing the spreading dynamics of social contagion on random networks. Agent-based simulations and theoretical analyses have revealed the coexistence of both continuous and discontinuous phase transitions in ER random networks. Study of two empirical networks with a heterogeneous degree distribution and a substantial amount of clustering has indicated that only continuous transitions can arise in such networks, and we have provided a physical understanding. Our findings are consistent with previous results from threshold models incorporating some kind of social reinforcement mechanism [13,16,40]. The physical origin of the discontinuous transition in a social system with a large number of close contacts can be attributed to their abundance through a drastic avalanche process $[13,34]$. A general result is that the value of the outbreak threshold is mainly determined by the abundance of the close contacts.

For ER networks, our study has revealed that close social contacts alone are not capable of triggering a large-scale outbreak of contagion. To have a global outbreak, a sufficient amount of ordinary transmission is needed. We have obtained a physical understanding of this phenomenon through a detailed statistical analysis of the spreading dynamics in terms of the frequencies of three different types of transmission 

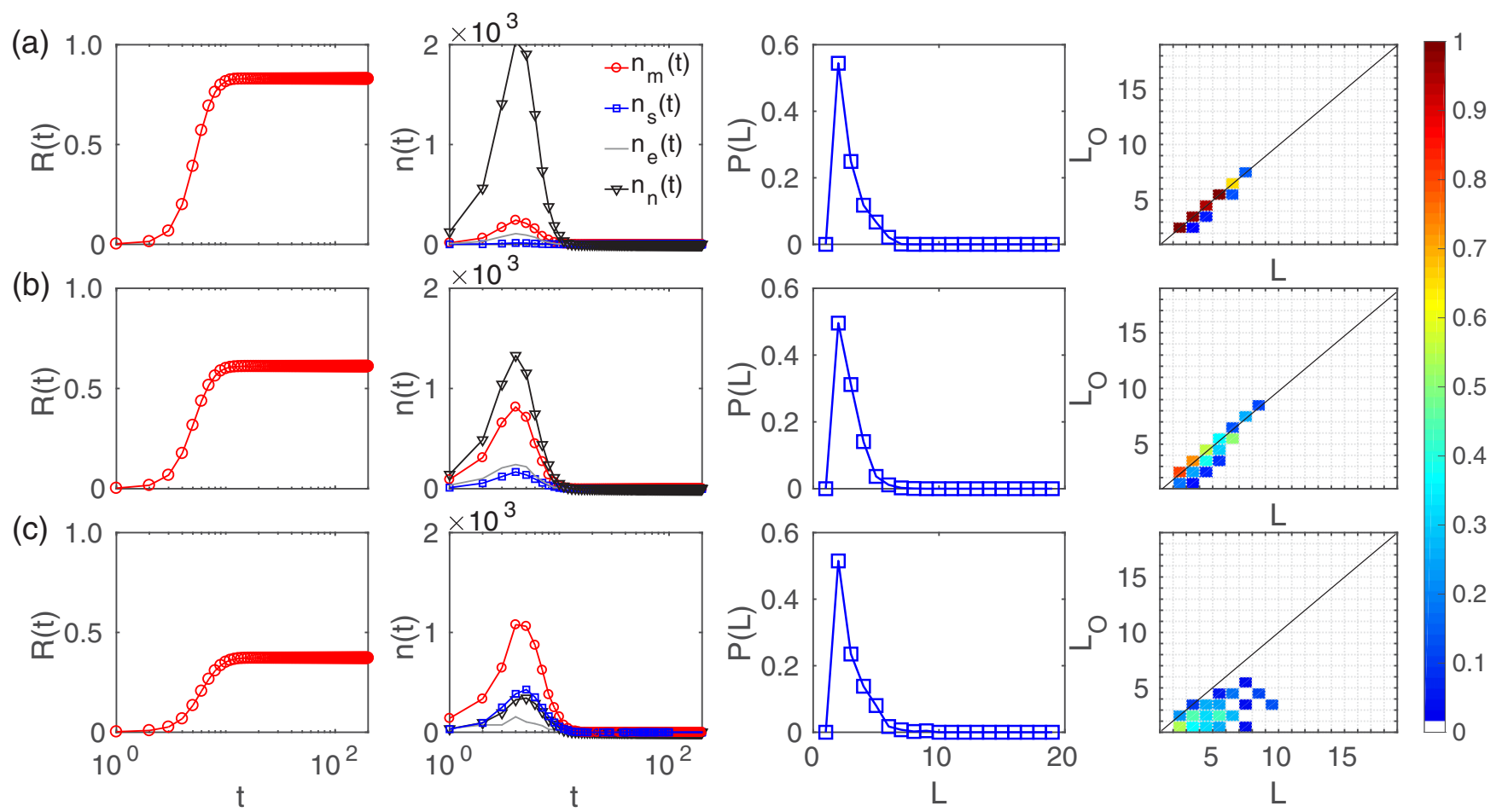

FIG. 15. The roles of two types of contacts on spreading dynamics in the PGP network-additional support. Legends are the same as in Fig. 14 except for $p_{h}<p=0.9$.

modes, the distribution of diffusion paths of various lengths, and the frequencies of the occurrence of transmission along the diffusion paths. The analysis has revealed that, to achieve maximum spreading, a matching condition must be met: $n_{O}(t) \approx n_{D}(t)$, i.e., the number of close contacts available to susceptible individuals in the critical state must approximately match the number of ordinary contacts. There exists an optimal fraction of close contacts to maximize the spreading, which depends on the values of the transmission rate and adoption threshold. The key to making spreading prevalent lies in the interplay between ordinary and successfully reinforced transmission events associated with long diffusion paths, which cannot occur unless there is a sufficient amount of ordinary transmission. If ordinary contacts are scarce, then spreading will become stagnant. Ordinary contacts thus play a crucial role in promoting social contagion when both types of social contacts are simultaneously present. In addition, a sufficient number of close contacts can change the nature of the phase transition from continuous to discontinuous, and leads to an increase in the value of the outbreak threshold.

For heterogeneous networks, a different picture of contagion spreading dynamics arises. While ordinary contacts still play a dominating role in the global prevalence of the contagion, there is a sensitivity to the threshold required for a successful adoption along close contacts. In particular, for a small threshold value, abundant close contacts facilitate the outbreak due to the availability of the transmission channels among the hub nodes, regardless of the value of the transmission rate. Close contacts are capable of promoting spreading but only on a local scale, especially near the hubs, making it difficult to achieve global prevalence of the contagion even though contagion outbreaks can still occur. For a large threshold value, close contacts tend to become an obstacle to transmission: global contagion will be prevented if the network is rich in close contacts, which is similar to what happens in ER networks. The lack of avalanche-like spreading, which results from inadequate number of susceptible individuals in the critical state, imposes a limit to the cooperation between ordinary and successful reinforced transmission induced by two types of contacts along long paths, leading to the absence of an optimal intermediate value $\mu$ to maximize the contagion spreading.

We conclude our work by providing two general remarks. First, numerical computations are inadequate to conclude the coexistence of continuous and discontinuous transitions in ER networks. Nonetheless, the issue for ER networks can be settled analytically through a comprehensive bifurcation analysis of the system dynamics using theoretical methods such as the edge-based compartmental approach [33], which we have carried out. Second, the main goal of our present study is to develop a computational and theoretical paradigm to understand the relative roles played by ordinary and close contacts in the social contagion. For this reason, we have studied the process involving a single contagion for both static random and empirical heterogeneous networks. We hope to be able to extend our analysis of either coupled spreading scenarios [41] or contagion dynamics to more diverse complex networks, as well as to temporal networks [42], multilayer networks [43-47], and metapopulation systems [48].

\section{ACKNOWLEDGMENTS}

This work was partially supported by China Postdoctoral Science Foundation (PSF) under Grants No. 2015M582532 
and No. 2018M631073, by Fundamental Research Funds for the Central Universities (Grant No. YJ201830), by Science Strength Promotion Programme of UESTC and by the National Natural Science Foundation of China under Grants No. 61673086 and No. 61433014. Y.C.L. acknowledges support from the Vannevar Bush Faculty Fellowship program sponsored by the Basic Research Office of the Assistant Secretary of Defense for Research and Engineering and funded by the Office of Naval Research through Grant No. N00014-16-12828 .
[1] D. J. Watts, Proc. Natl. Acad. Sci. U.S.A. 99, 5766 (2002).

[2] P. S. Dodds and D. J. Watts, Phys. Rev. Lett. 92, 218701 (2004).

[3] D. Centola and M. Macy, Am. J. Sociol. 113, 702 (2007).

[4] O. Yağan and V. Gligor, Phys. Rev. E 86, 036103 (2012).

[5] C. H. Weiss, J. Poncela-Casasnovas, J. I. Glaser, A. R. Pah, S. D. Persell, D. W. Baker, R. G. Wunderink, and L. A. Nunes Amaral, Phys. Rev. X 4, 041008 (2014).

[6] A. Nematzadeh, E. Ferrara, A. Flammini, and Y.-Y. Ahn, Phys. Rev. Lett. 113, 088701 (2014).

[7] K.-M. Lee, C. D. Brummitt, and K.-I. Goh, Phys. Rev. E 90, 062816 (2014).

[8] M. Karsai, G. Iniguez, K. Kaski, and J. Kertész, J. Royal Soc. Interface 11, 20140694 (2014).

[9] D. Centola, How Behavior Spreads: The Science of Complex Contagions (Princeton University Press, Princeton, NJ, 2018).

[10] D. Guilbeault, J. Becker, and D. Centola, in Complex Spreading Phenomena in Social Systems, edited by S. Lehmann and Y. Y. Ahn (Springer, Berlin, 2018), pp. 3-25.

[11] Z. Ruan, G. Iniguez, M. Karsai, and J. Kertész, Phys. Rev. Lett. 115, 218702 (2015).

[12] P. Cui, M. Tang, and Z.-X. Wu, Sci. Rep. 4, 6303 (2014).

[13] W. Wang, M. Tang, H.-F. Zhang, and Y.-C. Lai, Phys. Rev. E 92, 012820 (2015).

[14] A. Barrat, M. Barthelemy, and A. Vespignani, in Large Scale Structure And Dynamics Of Complex Networks: From Information Technology to Finance and Natural Science, edited by C. Caldarelli (World Scientific, Singapore, 2007), pp. 67-92.

[15] M. Karsai, G. Iñiguez, R. Kikas, K. Kaski, and J. Kertész, Sci. Rep. 6, 27178 (2016).

[16] Y.-X. Zhu, W. Wang, M. Tang, and Y.-Y. Ahn, Phys. Rev. E 96, 012306 (2017).

[17] D. Centola, Science 329, 1194 (2010).

[18] L. Lü, D.-B. Chen, and T. Zhou, New J. Phys. 13, 123005 (2011).

[19] P. Piedrahita, J. Borge-Holthoefer, Y. Moreno, and A. Arenas, Europhys. Lett. 104, 48004 (2013).

[20] N. O. Hodas and K. Lerman, Sci. Rep. 4, 4343 (2014).

[21] P. F. Lazarsfeld and R. K. Merton, in The Communication of Ideas: A Series of Addresses, edited by L. Bryson (Institute for Religious and Social Studies, New York, 1948), pp. 95-118.

[22] S. H. Chaffee and D. C. Mutz, in Advancing Communication Science: Merging Mass and Interpersonal Processes, edited by R. P. H. et al. (SAGE Publications, Thousand Oaks, CA, 1988), pp. $19-43$.

[23] D. A. Scheufele, Commun. Res. 29, 46 (2002).
[24] P. Desmet, J. van Spanje, and C. H. de Vreese, Int. J. Commun. 9, 3177 (2015).

[25] T. R. Vollmer and T. D. Hackenberg, J. Appl. Behav. Anal. 34, 241 (2001).

[26] W. D. Pierce and C. D. Cheney, Behavior Analysis and Learning (Psychology Press, London, UK, 2013).

[27] B. F. Skinner and C. Ferster, Schedules of Reinforcement (BF Skinner Foundation, Cambridge, MA, USA, 2015).

[28] J. C. Miller, A. C. Slim, and E. M. Volz, J. R. Soc. Interface 9, 890 (2012).

[29] J. C. Miller and E. M. Volz, PloS One 8, e69162 (2013).

[30] J. C. Miller and I. Z. Kiss, Math. Model. Nat. Phenom. 9, 4 (2014).

[31] W. Wang, M. Tang, H. E. Stanley, and L. A. Braunstein, Rep. Prog. Phys. 80, 036603 (2017).

[32] B. Karrer and M. E. J. Newman, Phys. Rev. E 82, 016101 (2010).

[33] R. Parshani, S. V. Buldyrev, and S. Havlin, Phys. Rev. Lett. 105, 048701 (2010).

[34] P.-B. Cui, L. Gao, and W. Wang, J. Theor. Biol. 441, 19 (2018).

[35] M. Ausloos and F. Petroni, arXiv:1401.4270.

[36] D. S. Callaway, M. E. J. Newman, S. H. Strogatz, and D. J. Watts, Phys. Rev. Lett. 85, 5468 (2000).

[37] R. Pastor-Satorras, C. Castellano, P. Van Mieghem, and A. Vespignani, Rev. Mod. Phys. 87, 925 (2015).

[38] M. Boguñá, R. Pastor-Satorras, A. Díaz-Guilera, and A. Arenas, Phys. Rev. E 70, 056122 (2004).

[39] E. Cho, S. A. Myers, and J. Leskovec, in Proceedings of the 17th ACM SIGKDD International Conference on Knowledge Discovery and Data Mining (ACM, New York, 2011) pp. 1082-1090.

[40] W. Cai, L. Chen, F. Ghanbarnejad, and P. Grassberger, Nature Phys. 11, 936 (2015).

[41] P.-B. Cui, F. Colaiori, and C. Castellano, Phys. Rev. E 96, 022301 (2017).

[42] P. Holme and J. Saramäki, Phys. Rep. 519, 97 (2012).

[43] M. Kivelä, A. Arenas, M. Barthelemy, J. P. Gleeson, Y. Moreno, and M. A. Porter, J. Comp. Net. 2, 203 (2014).

[44] S. Boccaletti, G. Bianconi, R. Criado, C. I. Del Genio, J. Gómez-Gardenes, M. Romance, I. Sendina-Nadal, Z. Wang, and M. Zanin, Phys. Rep. 544, 1 (2014).

[45] F. Battiston, M. Perc, and V. Latora, New J. Phys. 19, 073017 (2017).

[46] X. Chen, W. Wang, S. Cai, H. E. Stanley, and L. A. Braunstein, J. Stat. Mech. Theory Exp. 2018, 053501 (2018).

[47] Q.-H. Liu, W. Wang, S.-M. Cai, M. Tang, and Y.-C. Lai, Phys. Rev. E 97, 022311 (2018).

[48] L. Wang and J. T. Wu, Nat. Commun. 9, 218 (2018). 